The Astrophysical Journal, 463:498-508, 1996 June 1

(C) 1996. The American Astronomical Society. All rights reserved. Printed in U.S.A.

\title{
A COMPARISON BETWEEN THE NARROW-LINE REGIONS OF SEYFERT 1
} AND SEYFERT 2 GALAXIES ${ }^{1}$

\author{
HENRIQUE R. SCHMITT ${ }^{2,3}$ \\ Space Telescope Science Institute; and Depto. de Astronomia, IF-UFRGS, CP15051, CEP91501-970, Porto Alegre, RS, Brazil \\ AND \\ ANNE L. KINNEY ${ }^{4}$ \\ Space Telescope Science Institute, 3700 San Martin Drive, Baltimore, MD 21218 \\ Received 1995 August 28; accepted 1995 December 20
}

\begin{abstract}
We present high-resolution narrow-band [O III] images of Seyfert 1 and Seyfert 2 galaxies, obtained in the Hubble Space Telescope Data Archive. The continuum-subtracted [O III] images were used to measure the narrow-line region (NLR) sizes and compare them with the predictions from the Unified Scheme. This paper shows that the Seyfert 1 NLR sizes are much smaller than the NLR sizes of the Seyfert 2's if they were observed pole-on. We propose that the Unified Scheme is not wrong but rather that the Seyfert 1's may have their torus axes aligned close to the galaxy plane axis.

Subject headings: galaxies: active — galaxies: Seyfert — galaxies: structure
\end{abstract}

\section{INTRODUCTION}

The Unified Scheme for active galactic nuclei (AGNs) is based on the idea that the nuclear engine is surrounded by a dusty molecular torus, with orientation angle being the parameter determining whether an AGN is perceived by observers on Earth as a Seyfert 1 or as a Seyfert 2 (see Antonucci 1993 and Urry \& Padovani 1995 for a review of the model). This scenario is supported by the observation of hidden broad-line regions (BLRs) visible in polarized light (Antonucci \& Miller 1985; Miller \& Goodrich 1990), by a deficit of ionizing photons (Wilson, Ward, \& Haniff 1988; Kinney et al. 1991), and by the escape of collimated radiation from the nuclear region, which can be observed as jetlike radio structures (Ulvestad \& Wilson 1984a, b, 1989) and conical or biconical narrow-line regions (NLRs) in Seyfert 2 galaxies (Pogge 1988a, b, 1989; Schmitt, StorchiBergmann, \& Baldwin 1994; Schmitt \& Storchi-Bergmann 1995 and references therein).

The easiest observable evidence for the Unified Scheme is the collimation of nuclear radiation. This effect is presented in Figure 1 in which we show a cartoon representing the NLR as seen from different inclination angles. On the left we see the classical Seyfert 2 galaxy, with the nucleus covered by the torus (not represented here for reasons of clarity) and a cone-shaped NLR. We also introduce here the quantity " $d$," which is defined as the maximum extension of the cone, and the quantity " $b$," which is defined as the cone's base extension, the extension the NLR would have if it were observed pole-on, like a Seyfert 1 . In the middle we show an intermediate case between a Seyfert 1 and a Seyfert 2 galaxy, in which we can see that the cone-shaped NLR is still visible. Finally, on the right we show the Seyfert 1 case, in which the NLR should be seen as a halo. If the difference between Seyfert 2's and Seyfert 1's is only the orientation

\footnotetext{
${ }^{1}$ Based on observations made with the NASA/ESA Hubble Space Telescope, obtained from the data archive at the Space Telescope Science Institute. STScI is operated by the Association of Universities for Research in Astronomy, Inc. under NASA contract NAS 5-26555.

${ }^{2}$ CNPq Fellow.

3 schmitt@stsci.edu.

${ }^{4}$ kinney@stsci.edu.
}

angle of the line of sight to the object, the NLR should be visible in both Seyfert 2's and Seyfert 1's, and both should be of roughly comparable size, as illustrated in Figure 1. Note that we make the simplistic assumption that all NLRs have common properties such as density, central ionizing luminosity, and volume-filling factor.

The NLR shape as predicted by the Unified Scheme has been addressed by Pogge (1989). Using ground-based data, Pogge (1989) attempted to resolve the NLRs of Seyfert 1 galaxies. Although his images had low spatial resolution, he found that in a sample of nine nearby Seyfert 1's, six had unresolved, pointlike nuclear emission, and only three presented extended emission, which apparently contradicts the Unified Scheme.

In this paper we compare high-resolution [O III] images of Seyfert 1 and Seyfert 2 galaxies, available in the Hubble Space Telescope (HST) data archive, with the idealized picture of the Unified Scheme given in Figure 1. We find that the sizes of the NLRs of Seyfert 2 galaxies, measured as if we were looking at them from the same orientation as the Seyfert 1 galaxies, are much larger than the NLRs of the Seyfert 1's, which is similar to the result obtained by Pogge (1989). We propose that this result does not invalidate the Unified Scheme but rather indicates that there is another parameter to be taken into account.

In $\S 2$ we describe the observations and reductions. In $\S 3$ we discuss the selection effects and describe the method used to compare the two groups. In $\S 4$ we give the results and discuss their implications to the Unified Scheme. In $\S 5$ we give the conclusions, and in the Appendix we describe the galaxies.

\section{DATA AND REDUCTIONS}

The observations were taken from the HST data archive. We include observations of eight Seyfert 1's and 12 Seyfert 2's, out of a total of 10 Seyfert 1's and 15 Seyfert 2's in the archive. The excluded galaxies (NGC 2992, NGC 5506, NGC 5548, IC 5063, and Akn 120) were either saturated in the nucleus or placed in the field in such a way that the NLR was not included in the image. The images were obtained with the Faint Object Camera (FOC) after the COSTAR deployment and the Wide Field Planetary 

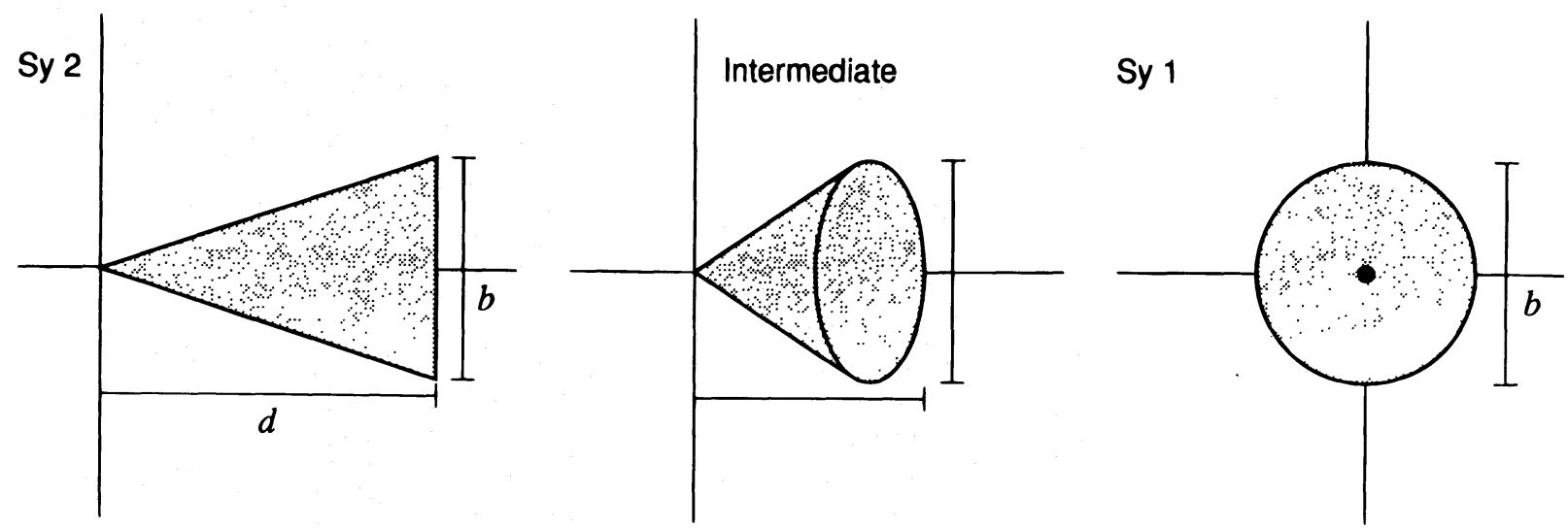

FIG. 1.-One cone of the narrow-line region. On the left, the cone is shown edge-on, with the nucleus at its apex. In the middle, the NLR cone is shown at an intermediate angle. On the right-hand side, the NLR cone is shown face-on with a small dot representing the broad-line region. An extended narrow-line region should be readily observable from any viewing angle.

Camera 1 (WF/PC 1), using filters centered in the redshifted line [O III] $\lambda 5007$ and/or [O III] $\lambda 4959$ and the adjacent continuum. We use [O III] images, because they trace the high-excitation gas and are less sensitive to $\mathrm{H}$ II regions than, for example, $H \alpha$. In Table 1 we present the observed objects, their activity class (Seyfert 1 or Seyfert 2), the date of the observation, the root name of the file in the archive, the detector used, the filter, the exposure time, and the emission lines transmitted by the filter. For some of the galaxies in our sample, the [O III] images were obtained through a medium-band filter that included both [O III] $\lambda \lambda 4959,5007$ and $\mathrm{H} \beta$. In these cases we used the curves of the filters and the emission-line fluxes given by Whittle (1992) to calculate the percentage of the total emission due to $\mathrm{H} \beta$, which was usually less than $10 \%$ and did not affect our results. In Table 2 we give the basic data of our sample.

The FOC observations were obtained in the $\mathrm{f} / 151$, $512 \times 512$ mode (the former $\mathrm{f} / 96$ mode; Jedrzejewski et al. 1994), which has a pixel size of $0{ }^{\prime \prime} 014 \times 00^{\prime \prime} 014$ and gives a field of view of about $7^{\prime \prime} \times 7^{\prime \prime}$. The data were corrected for geometric distortion using a grid of regularly spaced reseaux marks and divided by the normalized and geometrically corrected internal flat-field frames. The background was estimated from regions free of emission and was subtracted. Finally, the images were calibrated applying the standard conversion rates from counts to flux $\left(F_{\lambda}\right)$.

The WF/PC 1 observations were taken in the PC mode, which consists of four CCDs of $800 \times 800$ pixels of size $00^{\prime \prime} 043 \times 0.043$ and gives a field of view of about $66^{\prime \prime} \times 66^{\prime \prime}$. The images were reduced by the WF/PC 1 Routine Science Data Processing pipeline, in the following steps: the bias level was removed, the bias image was subtracted, the preflash image was scaled and subtracted, the dark image was scaled and subtracted, and the image was flat-fielded (MacKenty et al. 1992). No delta flats were applied to the data obtained between decontaminations, since a visual inspection showed that the decontamination did not significantly change the emission-line images' morphology. The remaining calibration steps include the removal of cosmic rays, subtraction of the background as estimated from regions of the CCD free from sources, and flux calibration applying the standard conversion rates from counts to flux $\left(F_{\lambda} ;\right.$ Sparks, Ritchie, \& MacKenty 1992).

In order to correct the images obtained with WF/PC 1 for the effects of spherical aberration, we deconvolved them using the Lucy algorithm (Lucy 1974). We employed model point-spread functions (PSFs), with the program TINY TIM (Krist 1992) instead of PSFs based on observed stars because the model gives an image of infinite signal-to-noise ratio and is as effective as the PSF based on observations. Since the PSF changes from filter to filter and for different regions of the chip, we created PSFs for each of the images, centered on the galaxy position. The images were deconvolved using 50 iterations of the algorithm, which gave a final $\chi^{2} \approx 1 \%$.

Finally, we created continuum-free images by subtracting the appropriate deconvolved continuum image from the deconvolved line plus continuum image. Unfortunately it was not possible to do this for UGC 3374, Mrk 463E, and Mrk 477. UGC 3374 contained too many deconvolution residuals, and the other two were observed with filters that included the emission lines in both continuum and emission-line filters. More details will be given in the Appendix. The estimated final resolution of the images is 0 ."1 before COSTAR and 0".05 after it.

\section{RESULTS}

\subsection{Selection Effects}

This study suffers from being based on a random sample chosen from an archive as opposed to being based on a carefully selected sample in which the objects are chosen so as to match the intrinsic properties of the host galaxies. To address the problem of selection effects, we must investigate the isotropic emission from the two types of galaxies. To do this, we use the [O $\mathrm{III}]$ emission obtained from largeaperture spectroscopic data from the literature (Table 2). While the [O $\mathrm{III}]$ emission is not perfectly isotropic, it is predominantly isotropic (Heckman 1995) and can be used as an approximate estimator of intrinsic luminosity.

The average $\log \left(F_{[\mathrm{O} \text { III }]} 25007\right)$ values are $-12.23 \pm 0.63$ ergs $\mathrm{cm}^{-2} \mathrm{~s}^{-1}$ and $-12.08 \pm 0.62 \mathrm{ergs} \mathrm{cm}^{-2} \mathrm{~s}^{-1}$ for the Seyfert 1's and the Seyfert 2's, respectively, which are consistent within the dispersion. Comparing the [O III] luminosities of the two samples we find that the average value for Seyfert 1's is $40.32 \pm 1.10 \mathrm{ergs} \mathrm{s}^{-1}$ and for Seyfert 2's is $41.53 \pm 0.81 \mathrm{ergs} \mathrm{s}^{-1}$. These results show that the Seyfert 2 galaxies have slightly higher luminosities, which is expected since Seyfert 2's are more difficult to detect because of their hidden BLR. We show on the left-hand panel of Figure 2 a 
TABLE 1

OBSERVED OBJECTS

\begin{tabular}{|c|c|c|c|c|c|c|c|}
\hline Object Name & $\begin{array}{l}\text { Activity } \\
\text { Class }\end{array}$ & $\begin{array}{l}\text { Observing } \\
\text { Dates }\end{array}$ & Root Name & Detector & Filter & $\begin{array}{l}\text { Exposure } \\
\text { Time }\end{array}$ & Comments \\
\hline NGC $1566 .$. & 1 & 1990 Oct 27 & W0DN0101T & PC & F502N & 900 & {$[\mathrm{O} \mathrm{III}] \lambda 5007$} \\
\hline NGC $1566 \ldots . .$. & 1 & 1990 Oct 27 & W0DN0104T & PC & F547M & 180 & Continuum \\
\hline NGC $3227 \ldots \ldots$. & 1 & $1992 \mathrm{Feb} 2$ & W0UB0101T & PC & F502M & 1800 & {$[\mathrm{O} \mathrm{III}] \lambda 5007$} \\
\hline NGC $3227 \ldots \ldots$ & 1 & 1992 Feb 2 & W0UB0102T & PC & F547M & 350 & Continuum \\
\hline NGC $3783 . . . .$. & 1 & 1991 Jun 20 & W0MW0502T & PC & F502N & 1800 & [O III] $\lambda 4959$ \\
\hline NGC $3783 \ldots \ldots$ & 1 & 1991 Jun 20 & W0MW0501T & PC & F547M & 350 & Continuum \\
\hline NGC $4051 \ldots \ldots$ & 1 & 1991 Jun 20 & W0MW0202T & PC & F502N & 1800 & [O III] $\lambda 5007$ \\
\hline NGC $4051 \ldots \ldots$ & 1 & 1991 Jun 20 & W0MW0201T & PC & F547M & 350 & Continuum \\
\hline NGC $4151 \ldots \ldots$ & 1 & 1991 Jun 19 & W0MW0402T & PC & F502N & 1608 & {$[\mathrm{O}$ III $] \lambda 5007$} \\
\hline NGC $4151 \ldots \ldots$ & 1 & 1991 Jun 19 & W0MW0403T & $\mathrm{PC}$ & F547M & 350 & Continuum \\
\hline NGC $4395 \ldots \ldots$ & 1 & 1992 Jul 18 & W0ZQ0304T & PC & F502N & 300 & [O III] $\lambda 5007$ \\
\hline NGC $4395 \ldots \ldots$ & 1 & 1992 Jul 18 & W0ZQ0301T & PC & F547M & 120 & Continuum \\
\hline NGC $6814 \ldots \ldots$ & 1 & 1992 Apr 19 & W0UB0301T & $\mathrm{PC}$ & F502N & 1800 & {$[\mathrm{O} \mathrm{III}] \lambda 5007$} \\
\hline NGC $6814 \ldots \ldots$ & 1 & 1992 Apr 19 & W0UB0302T & $\mathrm{PC}$ & F547M & 350 & Continuum \\
\hline UGC $3374 \ldots \ldots$ & 1 & 1992 Sep 25 & W1150305T & $\mathrm{PC}$ & F492M & 500 & {$[\mathrm{O} \mathrm{III}] \lambda \lambda 4959,5007+\mathrm{H} \beta$} \\
\hline UGC 3374 . & 1 & 1992 Sep 25 & W1150306T & PC & F547M & 300 & Continuum \\
\hline NGC $1068 \ldots \ldots$ & 2 & 1990 Aug 26 & W0CN0101T & PC & F502N & 900 & {$[\mathrm{O} \mathrm{III}] \lambda 5007$} \\
\hline NGC $1068 \ldots \ldots$ & 2 & 1990 Aug 26 & W0CN0101T & PC & F547M & 180 & Continuum \\
\hline NGC $2110 \ldots \ldots$ & 2 & $1992 \operatorname{Sep} 27$ & W1150201T & $\mathrm{PC}$ & F492M & 737 & {$[\mathrm{O} \mathrm{III}] \lambda \lambda 4959,5007+\mathrm{H} \beta$} \\
\hline NGC $2110 \ldots \ldots$ & 2 & 1992 Sep 27 & W1150202T & $\mathrm{PC}$ & F492M & 707 & {$[\mathrm{O} \mathrm{III}] \lambda \lambda 4959,5007+\mathrm{H} \beta$} \\
\hline NGC $2110 \ldots \ldots$ & 2 & 1992 Sep 27 & W1150203T & PC & F547M & 709 & Continuum \\
\hline NGC $3393 . . . .$. & 2 & 1993 Jun 7 & W1DB0405T & PC & F502N & 2000 & {$[\mathrm{O} \mathrm{III}] \lambda 4959$} \\
\hline NGC $3393 . . . .$. & 2 & 1993 Jun 7 & W1DB0406T & PC & F502N & 2000 & {$[\mathrm{O} \mathrm{III}] \lambda 4959$} \\
\hline NGC $3393 . . . .$. & 2 & 1993 Jun 7 & W1DB0407T & $\mathrm{PC}$ & $\mathrm{F} 502 \mathrm{~N}$ & 2000 & [O III $] \lambda 4959$ \\
\hline NGC $3393 \ldots \ldots$. & 2 & 1993 Jun 7 & W1DB0408T & PC & F502N & 2000 & [O III $] \lambda 4959$ \\
\hline NGC $3393 . . . .$. & 2 & 1993 Jun 7 & W1DB0409T & PC & F502N & 2000 & {$[\mathrm{O} \mathrm{III}] \lambda 4959$} \\
\hline NGC $3393 \ldots \ldots$ & 2 & 1993 Jun 7 & W1DB0401T & PC & F547M & 600 & Continuum \\
\hline NGC $5728 \ldots \ldots$ & 2 & 1992 Sep 4 & W1150401T & PC & F492M & 600 & {$[\mathrm{O} \mathrm{III}] \lambda \lambda 4959,5007+\mathrm{H} \beta$} \\
\hline NGC $5728 \ldots \ldots$ & 2 & 1992 Sep 4 & W1150402T & PC & F492M & 600 & {$[\mathrm{O} \mathrm{III}] \lambda \lambda 4959,5007+\mathrm{H} \beta$} \\
\hline NGC $5728 \ldots \ldots$ & 2 & 1992 Sep 4 & W1150403T & PC & F547M & 600 & Continuum \\
\hline NGC $5929 \ldots \ldots$ & 2 & 1992 Sep 27 & W1150501T & PC & F492M & 900 & {$[\mathrm{O}$ III] $\lambda \lambda 4959,5007+\mathrm{H} \beta$} \\
\hline NGC $5929 \ldots \ldots$. & 2 & 1992 Sep 27 & W1150502T & PC & F492M & 708 & {$[\mathrm{O}$ III $] \lambda \lambda 4959,5007+\mathrm{H} \beta$} \\
\hline NGC $5929 \ldots \ldots$ & 2 & 1992 Sep 27 & W1150503T & $\mathrm{PC}$ & F547M & 900 & Continuum \\
\hline Mrk $3 \ldots \ldots \ldots \ldots$ & 2 & 1994 Mar 20 & $\mathrm{X} 2580103 \mathrm{~T}$ & FOC & F502M & 750 & {$[\mathrm{O} \mathrm{III}] \lambda \lambda 4959,5007+\mathrm{H} \beta$} \\
\hline Mrk $3 \ldots \ldots \ldots$ & 2 & 1994 Mar 20 & $\mathrm{X} 2580104 \mathrm{~T}$ & FOC & F550M & 196 & Continuum \\
\hline Mrk $78 \ldots \ldots \ldots$ & 2 & 1994 Mar 19 & $\mathrm{X} 2580303 \mathrm{~T}$ & FOC & F502M & 801 & {$[\mathrm{O} \mathrm{III}] \lambda \lambda 4959,5007+\mathrm{H} \beta$} \\
\hline Mrk 78 & 2 & 1994 Mar 19 & $\mathrm{X} 2580304 \mathrm{~T}$ & FOC & F550M & 1196 & Continuum \\
\hline Mrk $348 \ldots \ldots \ldots$ & 2 & 1994 Feb 17 & $\mathrm{X} 2740401 \mathrm{~T}$ & FOC & F502M & 2129 & {$[\mathrm{O}$ III] $\lambda \lambda 4959,5007+\mathrm{H} \beta$} \\
\hline Mrk 348 . & 2 & 1994 Feb 17 & $\mathrm{X} 2740402 \mathrm{~T}$ & FOC & F550M & 1796 & Continuum \\
\hline Mrk $463 \mathrm{E} \ldots \ldots$ & 2 & 1992 Jul 6 & W0MW0A02T & PC & F517N & 1000 & {$[\mathrm{O} \mathrm{III}] \lambda 4959+\mathrm{H} \beta$} \\
\hline Mrk $463 \mathrm{E} \ldots \ldots$ & 2 & 1992 Jul 6 & W0MW0A01T & PC & F547M & 400 & [O III $] \lambda \lambda 4959,5007+\mathrm{H} \beta$ \\
\hline Mrk $477 \ldots \ldots \ldots$ & 2 & 1992 Jul 5 & W0MW0902T & PC & F517N & 1000 & {$[\mathrm{O}$ III $] \lambda \lambda 4959,5007$} \\
\hline Mrk $477 \ldots \ldots \ldots$ & 2 & 1992 Jul 5 & W0MW0901T & PC & F547M & 400 & [O III] $\lambda \lambda 4959,5007$ \\
\hline Mrk $477 \ldots \ldots \ldots$ & 2 & 1992 Nov 20 & W0Z10Q01T & PC & F785LP & 260 & Continuum \\
\hline Mrk $573 \ldots \ldots \ldots$ & 2 & 1994 Feb 9 & $\mathrm{X} 2580503 \mathrm{~T}$ & FOC & F502M & 896 & {$[\mathrm{O} \mathrm{III}] \lambda \lambda 4959,5007+\mathrm{H} \beta$} \\
\hline Mrk $573 \ldots \ldots \ldots$ & 2 & $1994 \mathrm{Feb} 9$ & $\mathrm{X} 2580504 \mathrm{~T}$ & FOC & F550M & 1301 & Continuum \\
\hline UGC $2456 \ldots \ldots$ & 2 & 1992 Nov 2 & W1150101T & PC & F492M & 600 & {$[\mathrm{O} \mathrm{III}] \lambda \lambda 4959,5007+\mathrm{H} \beta$} \\
\hline UGC $2456 \ldots . .$. & 2 & 1992 Nov 2 & W1150102T & PC & F492M & 600 & {$[\mathrm{O}$ III $] \lambda \lambda 4959,5007+\mathrm{H} \beta$} \\
\hline UGC $2456 \ldots \ldots$ & 2 & 1992 Nov 2 & $\mathrm{~W} 1150103 \mathrm{~T}$ & $\mathrm{PC}$ & F547M & 500 & Continuum \\
\hline
\end{tabular}

histogram of [O III] luminosities. The two groups have considerable overlap in luminosity, with the Seyfert 1's having a tail toward low luminosities and the Seyfert 2's toward high luminosities.

For a sample of galaxies at different distances, observed with different exposure times, we must also ask what the limit of detectability is, in order to ensure that the limit is comparable between the Seyfert 2 and the Seyfert 1 galaxies. The average distance to the Seyfert 2 galaxies is $75.7 \pm 59.4$ Mpc, while the average distance to the Seyfert 1 galaxies is $24.6 \pm 25.0 \mathrm{Mpc}$. The histogram on the middle panel of Figure 2 shows the distribution of distances. The histogram of the logarithm of the $3 \sigma$ detection limit luminosity is shown on the right-hand panel of Figure 2. We can see that Seyfert 1's have, on average, slightly deeper limits of detectability, $37.46 \pm 0.33 \mathrm{ergs} \mathrm{s}^{-1} \AA^{-1} \operatorname{arcsec}^{-2}$, than Seyfert 2's, $38.15 \pm 0.72 \operatorname{ergs~s}^{-1} \AA^{-1} \operatorname{arcsec}^{-2}$, so we should be able to detect the full size of the NLRs of the Seyfert 1's

The two groups of galaxies have similar intrinsic luminosities and were observed such that the detection limits of Seyfert 1's are comparable to those of the Seyfert 2's. These comparisons imply that our observations are not biased toward the detection of faint extended emission in highluminosity Seyfert 2 galaxies.

\subsection{The Comparison Method}

The comparison we make here is similar to that performed by Antonucci \& Ulvestad (1985) in their search for extended emission in blazars; their hypothesis was that blazars are identical to normal radio galaxies and radio quasars but that blazars are observed face-on. They tested this hypothesis by performing high-resolution VLA observations of blazars in search of their extended radio component. Antonucci \& Ulvestad were looking for the faint, 
TABLE 2

SAMPLe Properties

\begin{tabular}{|c|c|c|c|c|c|c|c|c|c|}
\hline Object $^{\mathrm{a}}$ & Morphological Type ${ }^{b}$ & Distance $^{c}$ & Scale $^{\mathrm{d}}$ & $\log \left(F_{[\mathrm{O} \text { III }}\right)^{\mathbf{e}}$ & $\log \left(L_{[\mathrm{O} \mathrm{IIII}}\right)^{\mathrm{f}}$ & $\mathbf{P A}^{\mathbf{g}}$ & $i^{\mathrm{h}}$ & $\log [F(\operatorname{Lim})]^{\mathrm{i}}$ & $\log [L(\operatorname{Lim})]^{\mathrm{j}}$ \\
\hline NGC $1566 \ldots . .$. & SAB(s)bc & 17.4 & 84.4 & -12.52 & 40.04 & 60 & 37 & 6.707 & 37.38 \\
\hline NGC $3227 \ldots \ldots$ & SAB(s)pec & 14.7 & 71.2 & -12.19 & 40.22 & 155 & 48 & 14.711 & 37.58 \\
\hline NGC $3783 \ldots .$. & $\left(\mathrm{R}^{\prime}\right) \mathrm{SB}(\mathrm{r}) \mathrm{ab}$ & 33.3 & 161.2 & -11.89 & 41.23 & $\approx$ fo & 26 & 4.705 & 37.79 \\
\hline NGC $4051 \ldots .$. & SAB(rs)bc & 10.0 & 48.2 & -12.41 & 39.67 & 135 & 41 & 12.331 & 37.17 \\
\hline NGC $4151 \ldots .$. & (R')SAB(rs)ab & 13.0 & 63.2 & -10.94 & 41.37 & 50 & 44 & 20.606 & 37.62 \\
\hline NGC $4395 \ldots .$. & $\mathrm{SA}(\mathrm{s}) \mathrm{m}$ & 4.1 & 19.6 & -12.90 & 38.40 & 147 & 33 & 43.591 & 36.94 \\
\hline NGC $6814 \ldots .$. & $\mathrm{SAB}(\mathrm{rs}) \mathrm{bc}$ & 21.9 & 106.2 & -12.87 & 39.90 & $\approx$ fo & 21 & 2.856 & 37.21 \\
\hline UGC $3374 \ldots \ldots$ & $\mathrm{S}$ & 82.6 & 400.2 & -12.15 & 41.76 & 90 & 44 & 1.190 & 37.99 \\
\hline Average ...... & $\cdots$ & $24.6 \pm 25.0$ & $\cdots$ & $-12.23 \pm 0.63$ & $40.32 \pm 1.10$ & $\cdots$ & $\ldots$ & $13.36 \pm 13.85$ & $37.46 \pm 0.33$ \\
\hline NGC $1068 \ldots .$. & (R)SA(rs)b & 16.5 & 79.8 & -10.70 & 41.81 & 70 & 32 & 4.813 & 37.19 \\
\hline NGC $2110 \ldots . .$. & SABO & 28.7 & 139.1 & -12.77 & 40.22 & 20 & 40 & 2.423 & 37.37 \\
\hline NGC $3393 \ldots . .$. & ( $\left.\mathrm{R}^{\prime}\right) \mathrm{SB}(\mathrm{rs}) \mathrm{a}$ & 47.1 & 228.3 & -11.90 & 41.52 & $\approx$ fo & 25 & 4.651 & 38.10 \\
\hline NGC $5728 \ldots \ldots$ & SAB(r)a & 37.3 & 180.8 & -12.17 & 41.05 & 0 & 55 & 1.422 & 37.37 \\
\hline NGC $5929 \ldots \ldots$ & Sab:pec & 35.8 & 173.6 & -13.03 & 40.16 & $\approx$ fo & 26 & 1.677 & 37.41 \\
\hline Mrk $3 \ldots \ldots \ldots$ & So & 55.0 & 266.6 & -11.46 & 42.10 & $\approx$ fo & 27 & 4.082 & 38.17 \\
\hline Mrk $78 \ldots \ldots \ldots$ & SB & 150.8 & 739.6 & -12.18 & 42.25 & $?$ & 60 & 3.571 & 38.99 \\
\hline Mrk $348 \ldots \ldots \ldots$ & $\mathrm{SA}(\mathrm{s}) 0 / \mathrm{a}$ & 62.3 & 301.8 & -12.38 & 41.29 & $\approx$ fo & 0 & 3.061 & 38.16 \\
\hline Mrk 463E ...... & Pair & 202.8 & 983.2 & -12.14 & 42.55 & Inter. & $\ldots$ & 5.625 & 39.44 \\
\hline Mrk $477 \ldots \ldots$. & Pair & 153.5 & 744.1 & -11.82 & 42.63 & Inter. & $\ldots$ & 5.949 & 39.22 \\
\hline Mrk $573 \ldots \ldots \ldots$ & (R)SAB(rs) $0+$ & 68.8 & 333.6 & -11.80 & 41.95 & $\approx$ fo & 0 & 4.082 & 38.36 \\
\hline UGC $2456 \ldots \ldots$ & (R)SB(s) $0+$ & 49.4 & 239.5 & -12.62 & 40.85 & 90 & 54 & 3.245 & 37.98 \\
\hline Average ...... & $\ldots$ & $75.7 \pm 59.4$ & $\ldots$ & $-12.08 \pm 0.62$ & $41.53 \pm 0.81$ & $\ldots$ & $\ldots$ & $3.72 \pm 1.38$ & $38.15 \pm 0.72$ \\
\hline
\end{tabular}

a Galaxy name.

${ }^{b}$ Morphological type taken from NED.

c Distance in Mpc, calculated using the radial velocity relative to the Local Group (de Vaucouleurs et al. 1991) and assuming $H_{0}=75 \mathrm{~km} \mathrm{~s}^{-1} \mathrm{Mpc}^{-1}$.

d Linear scale in pc arcsec -1 .

${ }^{\mathrm{e}}$ Logarithm of the [O $\left.\mathrm{III}\right] \lambda 5007 \AA$ flux in units of ergs $\mathrm{cm}^{-2} \mathrm{~s}^{-1}$. The data were taken from Whittle 1992 for most of the galaxies; from Díaz, Prieto, \& Wamsteker 1988 for NGC 3393; and from Filippenko, Ho, \& Sargent 1993 for NGC 4395.

${ }^{f}$ Logarithm of the [O III] $\lambda 5007 \AA$ luminosity in units of ergs s ${ }^{-1}$.

g Position angle of the galaxy's major axis (de Vaucouleurs et al. 1991). "Inter" means that the galaxy is an interacting system and " $\approx$ fo" means that the galaxy is approximately face-on.

${ }^{\mathrm{h}}$ Inclination of the galaxy disk relative to the plane of the sky, calculated from the relation between the major and the minor axis from de Vaucouleurs et al. 1991.

${ }^{i}$ Logarithm of the $3 \sigma$ detection limit surface brightness in units of $10^{-16} \mathrm{ergs} \mathrm{cm}^{-2} \mathrm{~s}^{-1} \AA^{-1} \operatorname{arcsec}^{-2}$, calculated from regions in the frame free from emission.

${ }^{j}$ Logarithm of the detection limit surface brightness corrected for the galaxy distance, in units of ergs s ${ }^{-1} \AA^{-1} \operatorname{arcsec}^{-2}$.

extended radio emission surrounding the very bright, compact, steep spectrum source of the blazars. While the aspect ratio of the radio jets (length to width) is different from the aspect ratio of the extended NLRs of the Seyfert 2 galaxies, the geometry is essentially the same; the emitting region appears elongated when observed edge-on and appears approximately circular when observed face-on, in spite of the additional bright, compact source present in the face-on case of a blazar or the bright, point source present in the face-on case of a Seyfert 1 galaxy, as illustrated in Figure 1.

The NLRs and the extended radio jets do have something in common besides geometry-these emission regions are both optically thin, isotropically emitting regions. Thus,

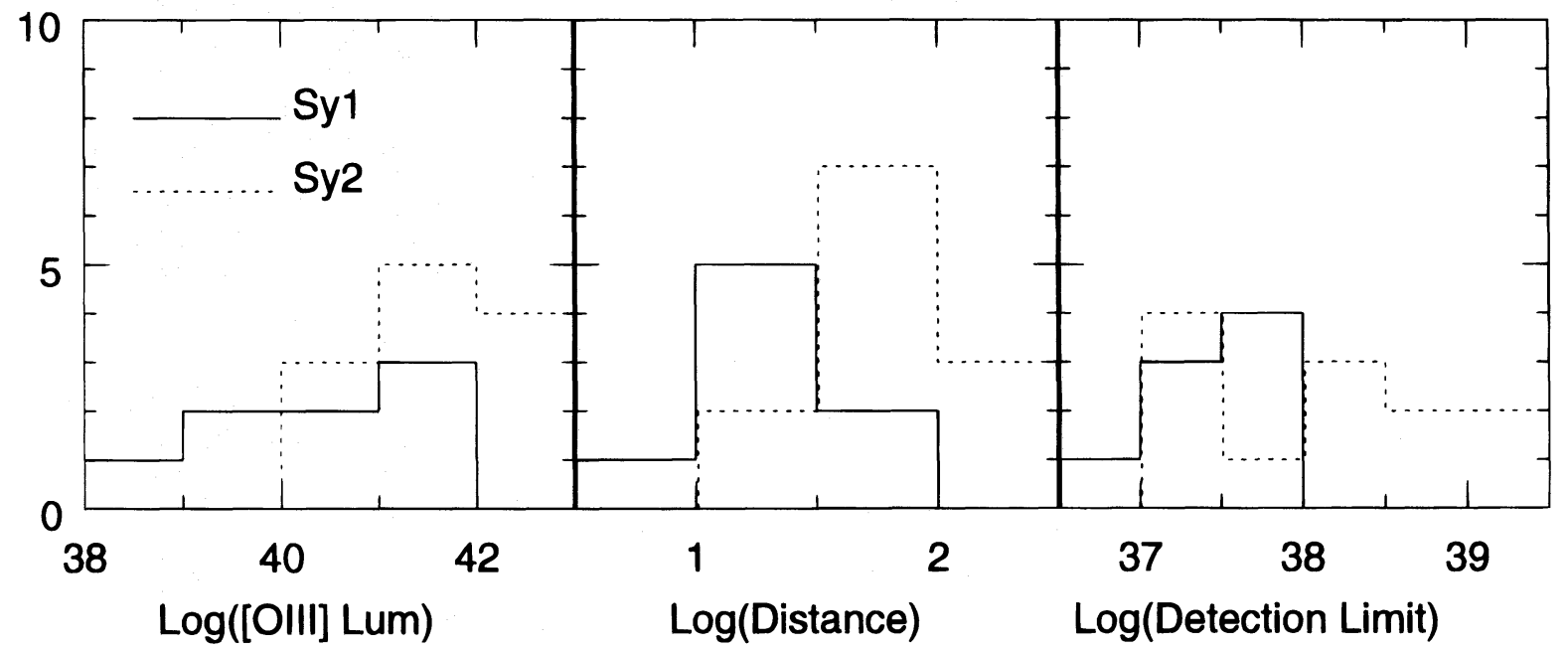

FIG. 2.-Left: Distribution of [O III] luminosities of the Seyfert 2 galaxies (dashed line) and the Seyfert 1 galaxies (solid line) as quoted in Whittle (1992). Middle: Distribution of the distances of the two groups. Right: Distribution of limit of detectability, expressed as a luminosity. 
they can be oriented in any direction on the sky, and, unless one's line of sight to the entire extended emitting region is obscured, the region will be detectable.

The method we use here and illustrate in Figure 3 consists of comparing the size of the Seyfert 1's NLRs with the size that the Seyfert 2's NLRs would have if they were observed pole-on, which corresponds to the extension " $b$ " shown in Figure 1. In order to perform this measurement and compare the two classes of galaxies, we create contour plots for each of the galaxies, where the lowest contour level corresponds to 3 times the standard deviation of the background $(3 \sigma)$.

On the left-hand panel of Figure 3 we give the example of a Seyfert 1 galaxy (NGC 1566). For the Seyfert 1's the NLR extension was computed by simply measuring the maximum extension of the emission over the $3 \sigma$ contour plot. Since these galaxies are observed pole-on, under the assumption of the Unified Scheme, and do not show conelike NLRs, this extension corresponds to the quantity " $b$," the cone base extension. For NGC 4151, which is known to have a conelike NLR, we measured the quantities " $d$ " and " $b$ " using the same method applied to the Seyfert 2's, described below. For most of the Seyfert 1's, the NLR was an unresolved, pointlike source, having no distinguishable structures. In these cases, the use of the $3 \sigma$ contour level for the measurement of " $b$ " provides a conservative measurement, representing an extension larger than the real NLR extension.

For Seyfert 2's, which are shown in the middle (UGC 2456) and right-hand (NGC 2110) panels of Figure 3, we use two different approaches to measure the NLR extensions, depending on morphology. In the first case, for the galaxies like UGC 2456, an emission-line cone or jetlike morphology can be clearly seen. For this object we superpose a cone by eye over the contour plots, assuming that the apex is given by the peak in the continuum image. The quantities " $d$," maximum extension of the emission, " $b$," the cone's base extension, as well as its opening angle are measured directly over the contours. These measurements were done in a conservative manner by including only the fully filled regions of the cone, as indicated in Figure 3. The second case we consider here is for galaxies such as NGC 2110, in which the NLR bends. In these cases we superpose a cone over the straight region of the NLR and also make the measurements considering only the filled parts of the cone.
Note that the measurements of the size of the NLRs were performed in such a way as to minimize the differences between the sizes of the NLRs of Seyfert 1's and those of Seyfert 2's. There is one case in our sample in which it was not possible to superpose a cone, Mrk 477, because of the intricate NLR of this galaxy and the residuals left by the deconvolution.

\section{DISCUSSION}

In Table 3 we present the most important properties of our sample (the maximum extension of the cone $[d]$, its opening angle, base extension $[b]$, and position angle of the extended emission) measured from the images. The emission-line images are shown in Figure 4 (Plates 34-40). We display them using the same linear dimension for all plates, with one side corresponding to $2 \mathrm{kpc}$. Mrk 463E and

TABLE 3

Measured Extensions

\begin{tabular}{|c|c|c|c|c|}
\hline Object Name & $\begin{array}{c}d^{\mathrm{a}} \\
(\mathrm{pc})\end{array}$ & $\begin{array}{l}\text { Opening } \\
\text { Angle }^{\mathrm{b}}\end{array}$ & $\begin{array}{l}b^{\mathrm{c}} \\
(\mathrm{pc})\end{array}$ & $\mathrm{PA}^{\mathrm{d}}$ \\
\hline NGC $1566 .$. & $\cdots$ & $\ldots$ & 59: & \\
\hline NGC $3227 \ldots$ & $\cdots$ & $\ldots$ & 100 & 15 \\
\hline NGC $3783 \ldots$ & $\ldots$ & $\ldots$ & 130: & \\
\hline NGC $4051 \ldots \ldots$ & $\cdots$ & & 58 & 100 \\
\hline NGC $4151 \ldots$ & 190 & 70 & 130 & 55 \\
\hline NGC $4395 .$. & $\ldots$ & $\ldots$ & 10 & \\
\hline NGC $6814 \ldots \ldots$ & $\ldots$ & $\ldots$ & 106: & 150 \\
\hline UGC $3374 \ldots \ldots$ & $\ldots$ & $\ldots$ & 160: & $\ldots$ \\
\hline NGC $1068 \ldots \ldots$ & 600 & 45 & 240 & 35 \\
\hline NGC $2110 \ldots \ldots$ & 140 & 35 & 56 & 345 \\
\hline NGC $3393 . . . .$. & 480 & 90 & 320 & 55 \\
\hline NGC $5728 \ldots \ldots$ & 720 & 70 & 560 & 120 \\
\hline NGC $5929 \ldots \ldots$ & 150 & 80 & 150 & 245 \\
\hline Mrk $3 \ldots \ldots \ldots \ldots$ & 280 & 45 & 175 & 70 \\
\hline Mrk 78 & 1500 & 45 & 500 & 70 \\
\hline Mrk 348 & 180 & 60 & 120 & 150 \\
\hline Mrk 463E & 885 & 40 & 490 & 180 \\
\hline Mrk $477 \ldots \ldots \ldots$ & 445 & & & 35 \\
\hline Mrk 573 & 670 & 70 & $600^{e}$ & 310 \\
\hline UGC $2456 \ldots \ldots$ & 300 & 45 & 120 & 310 \\
\hline
\end{tabular}

a Maximum extension of the emission

b Cone opening angle.

c Cone base extension; upper limits are indicated by a colon.

d Cone position angle.

${ }^{\mathrm{e}}$ Measured including the northwest blobs, as discussed in Appendix A.

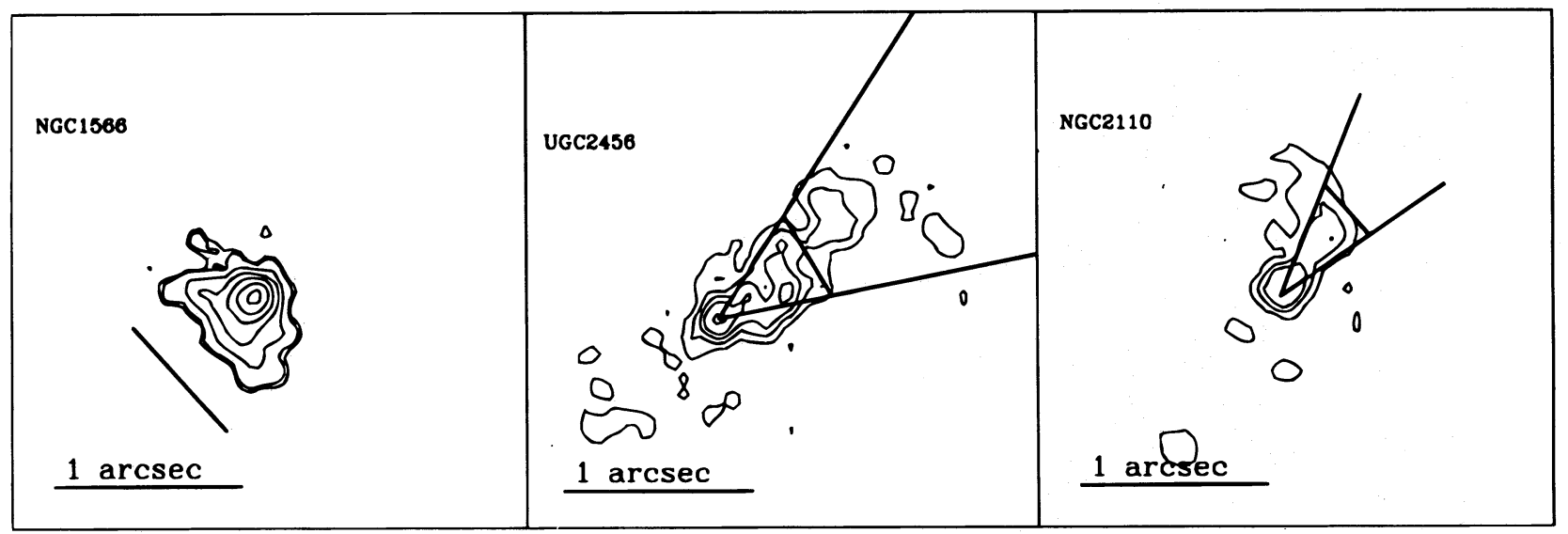

Fig. 3.-Examples of the method used to compare the NLR extension of the NLRs of Seyfert 1's (left) with the NLR extension of Seyfert 2's (middle and right) as if they were observed as Seyfert 1's. The lower contour level corresponds to $3 \sigma$ above the sky. 


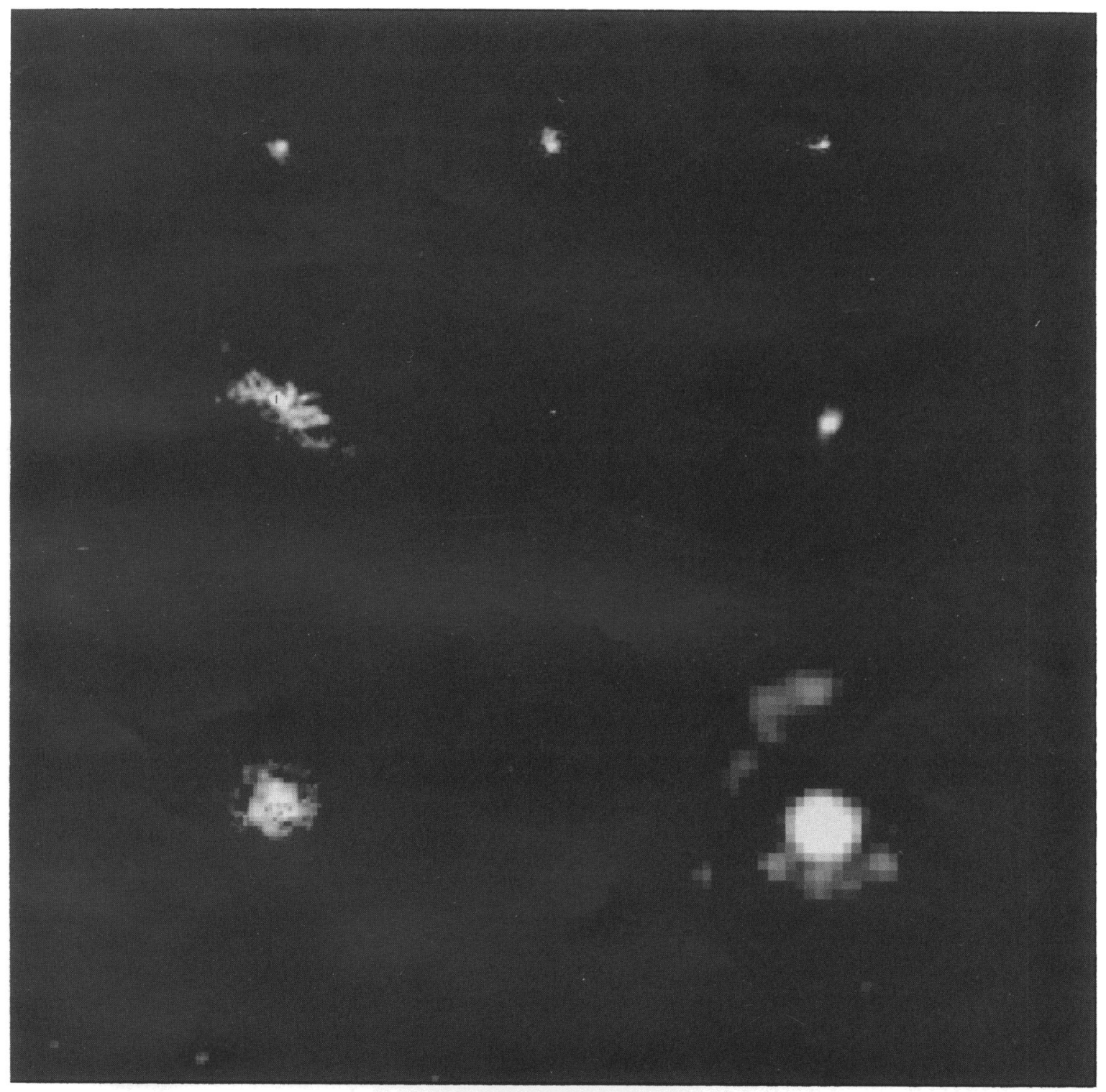

Fig. $4 a$

FIG. 4. - Gray-scale images of Seyfert 1 (Fig. $4 a$ ) and Seyfert 2 (Figs. $4 b-4 g$ ) galaxies. North is up, and east is to the left. The galaxies are all shown on the same spatial scale. Each plate is $2 \mathrm{kpc}$ on a side, except Fig. $4 f$ (Mrk 463E) and Fig. $4 g$ (Mrk 78), for which the side of the image corresponds to 4 kpc. Fig. $4 a$ : NGC 1566 (upper left), NGC 3227 (upper middle), NGC 4051 (upper right), NGC 4151 (middle left), NGC 4395 (middle), NGC 6814 (middle right), NGC 3783 (lower left), and UGC 3374 (lower right). Fig. 4b: Mrk 3 (upper left), NGC 1068 (upper right), NGC 2110 (lower left), and NGC 5929 (lower right). Fig. $4 c$ : Mrk 348 (upper left), UGC 2456 (upper right), Mrk 477 (lower left), and Mrk 573 (lower right). Fig. 4d: NGC 5728. Fig. 4e: NGC 3393. Fig. 4f: Mrk 78. Fig. 4g: Mrk 463E.

SchmitT \& KINNEY (see 463, 502) 
í

,

i:

II.

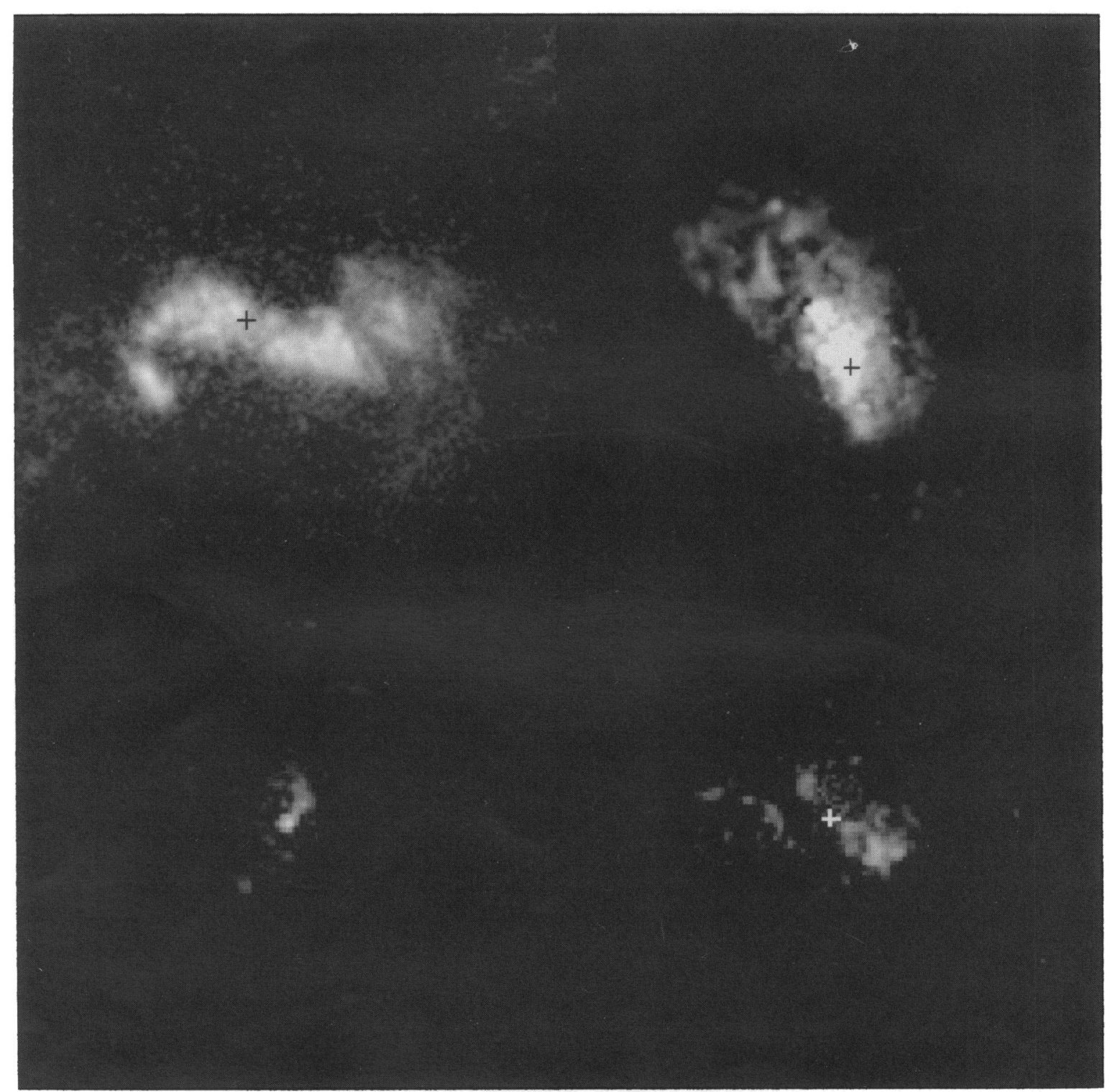

FIG. $4 b$

SCHMitT \& KinNEY (see 463, 502) 


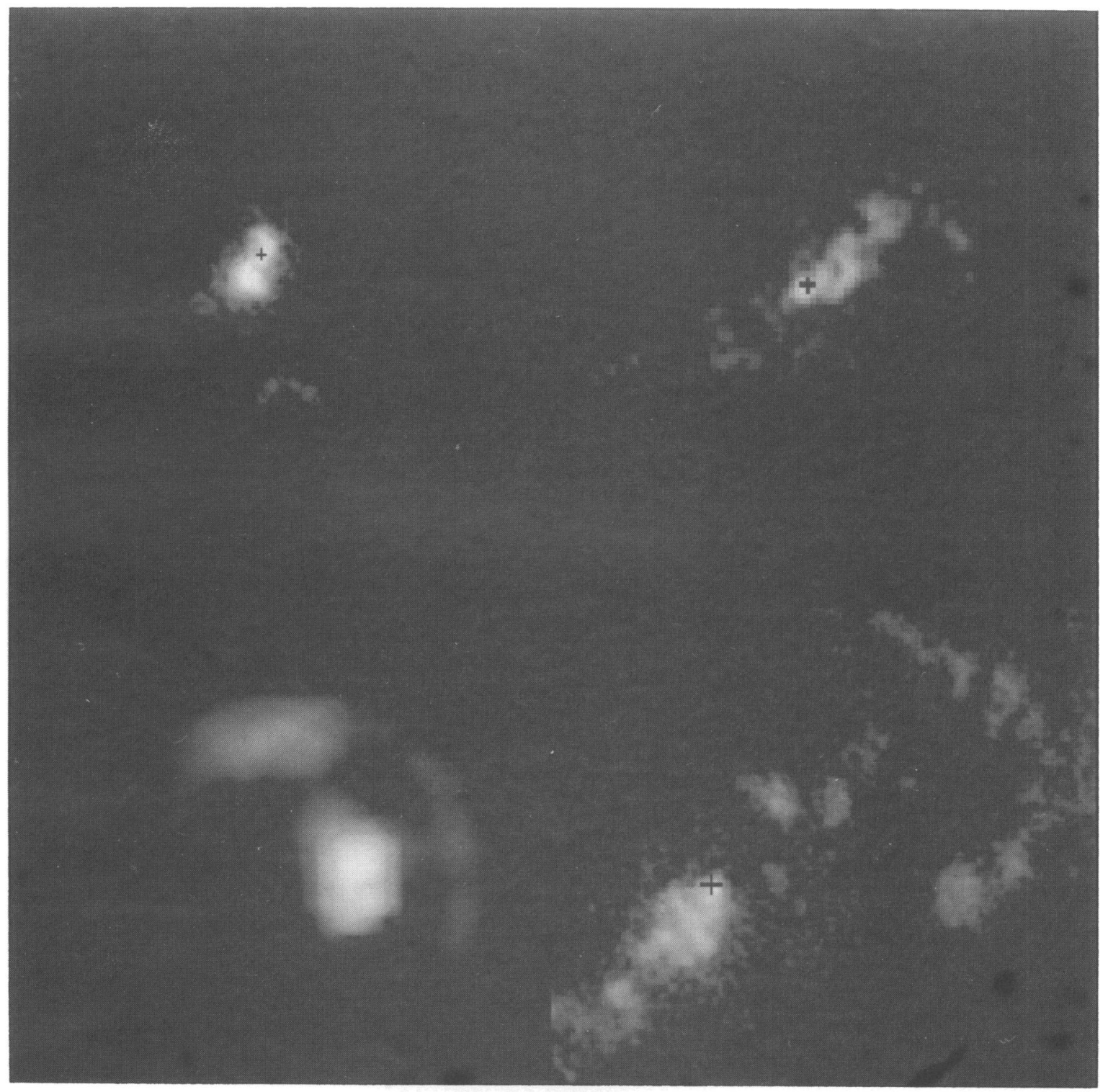

FIG. $4 c$

SCHMitT \& KINNEY (see 463, 502) 
101

10

1

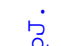

10

I,

InI

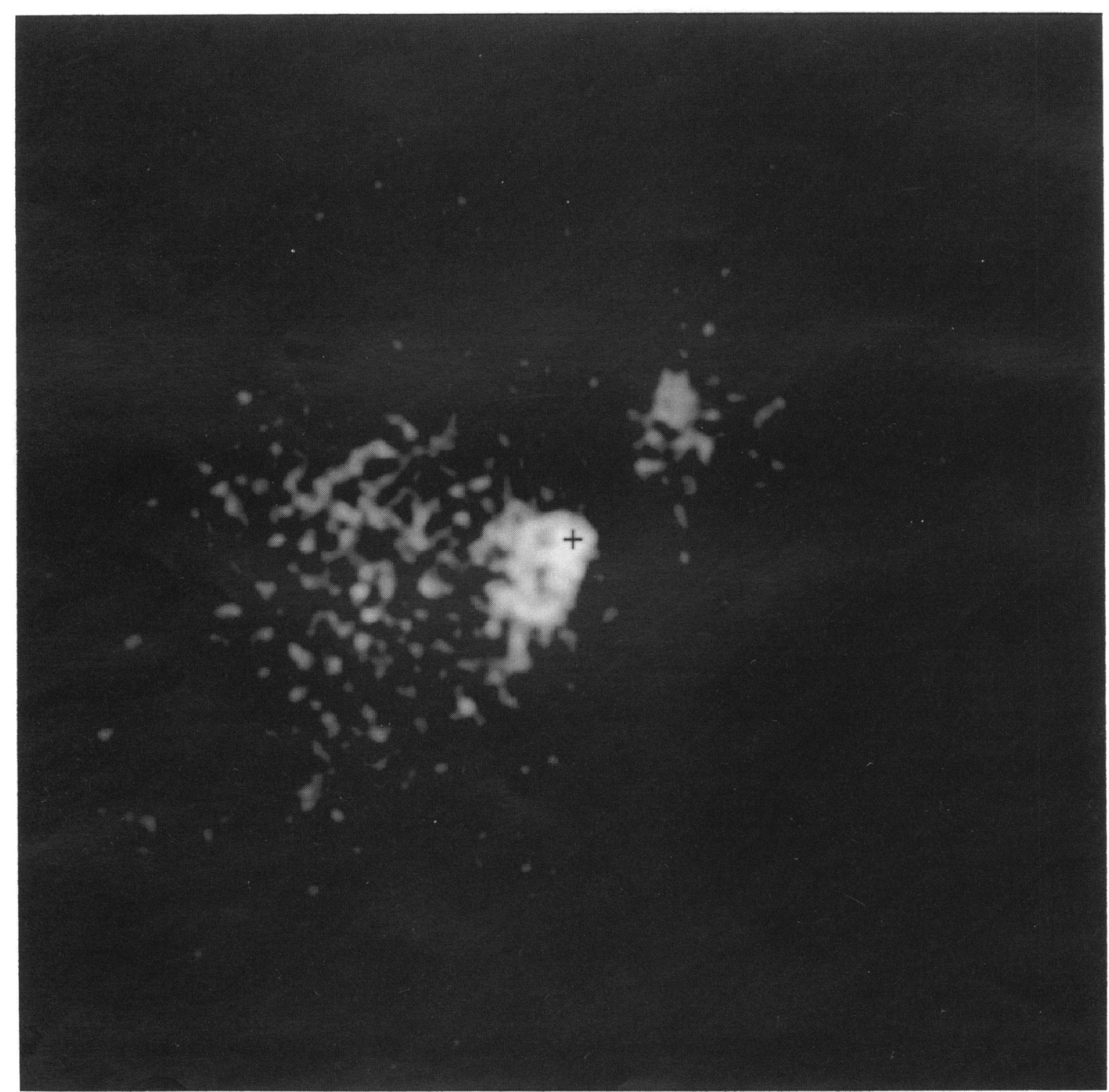

FIG. $4 d$

SCHMITT \& KINNEY (see 463, 502) 


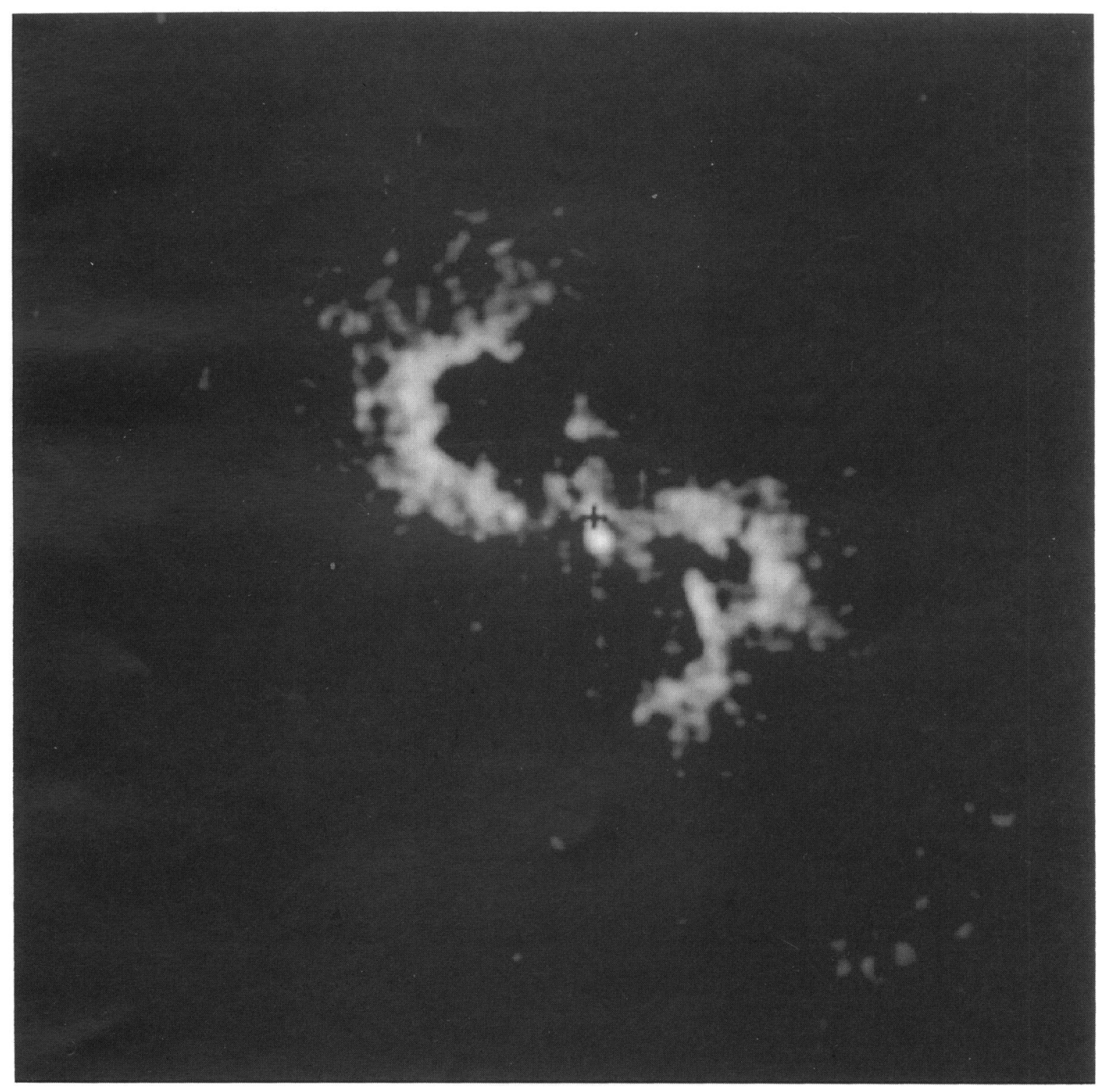

Fig. $4 e$

SChMITT \& KINNEY (see 463, 502) 


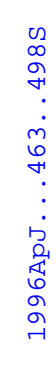

PLATE 39

I I I I

'm'

101

1

1

,

',

Iㄴ.

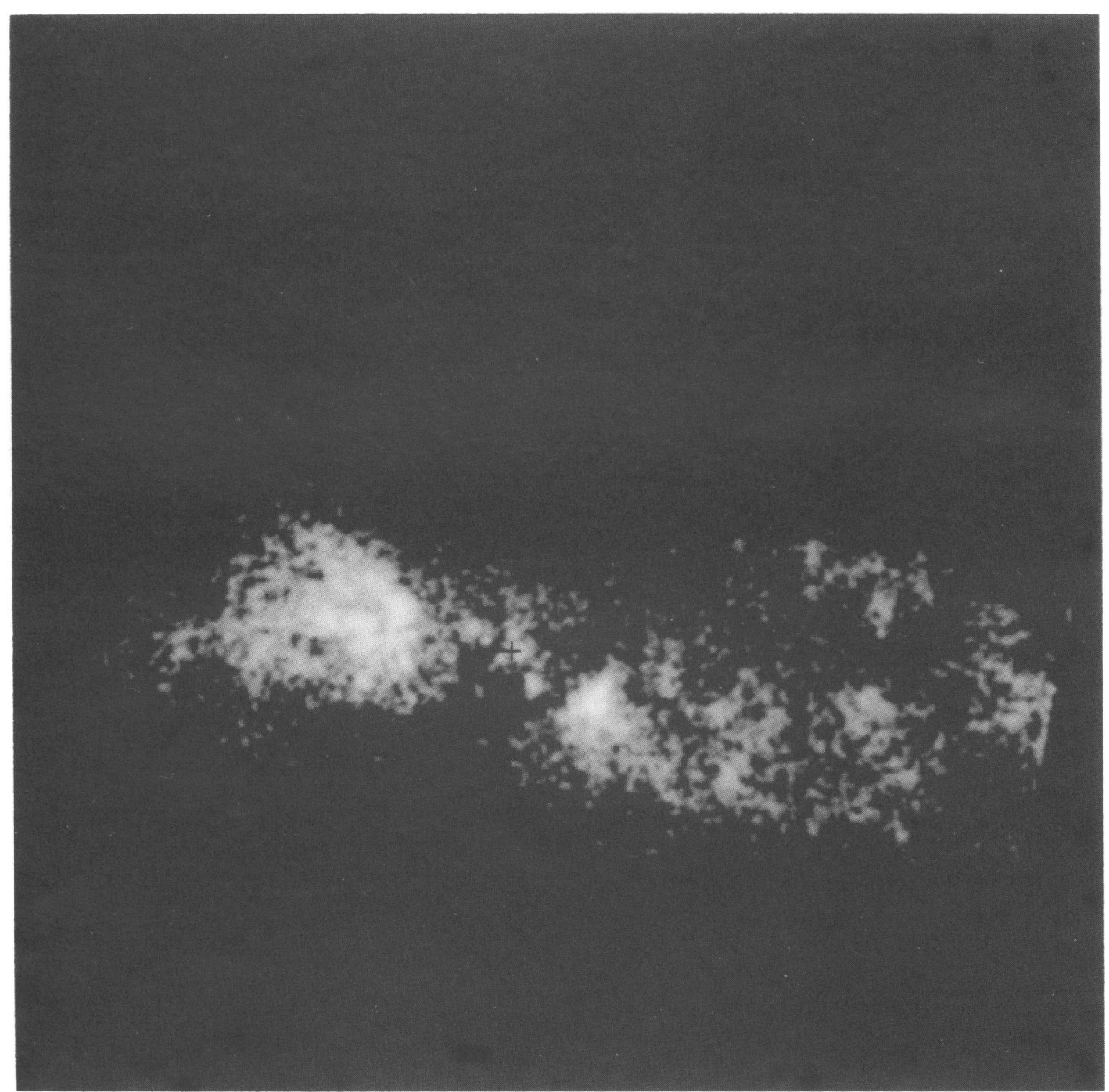

Fig. $4 f$

SChMitT \& KINNEY (see 463, 502)

(c) American Astronomical Society - Provided by the NASA Astrophysics Data System 


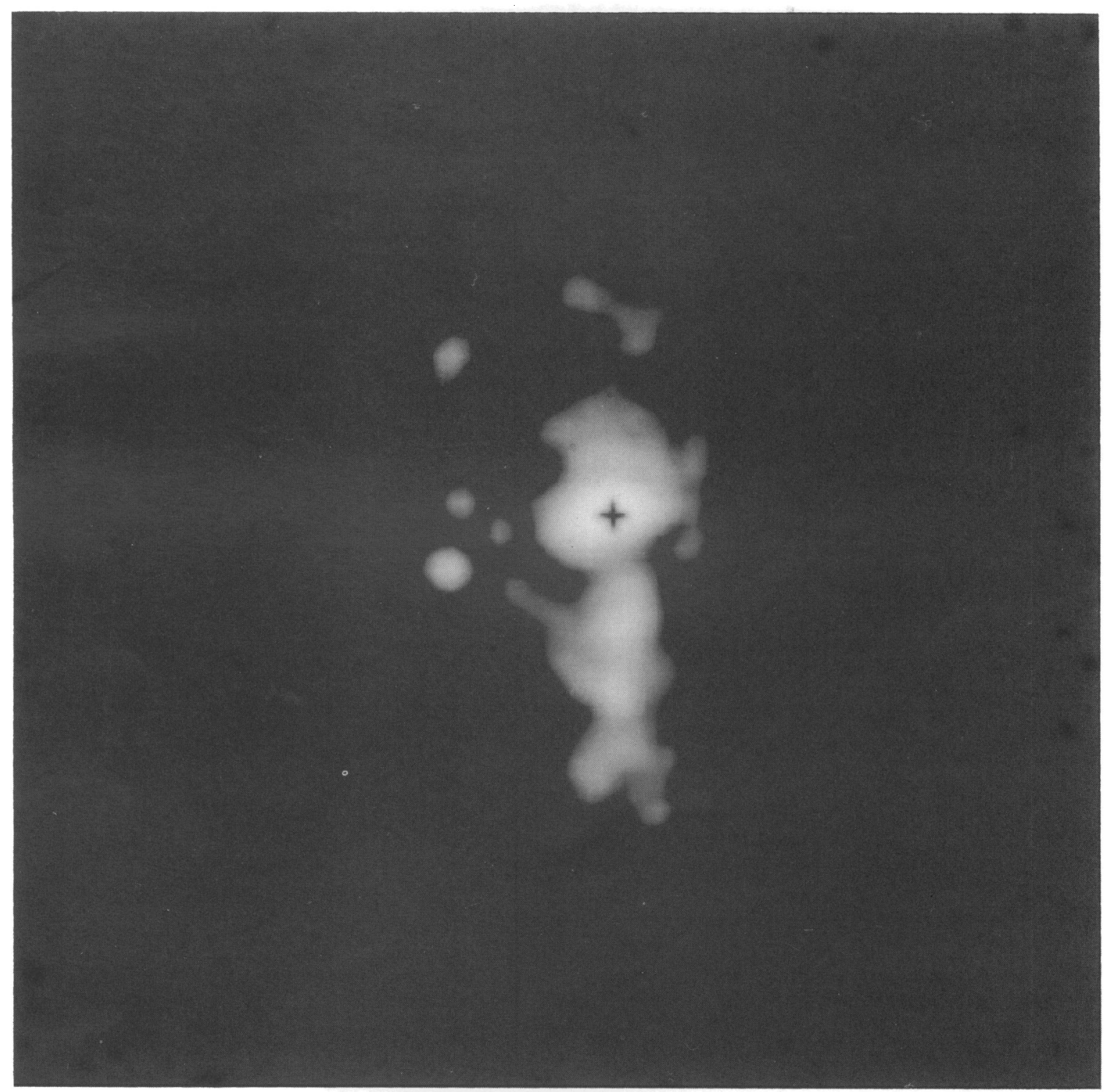

FIG. $4 g$

SCHMitT \& KINNEY (see 463, 502) 

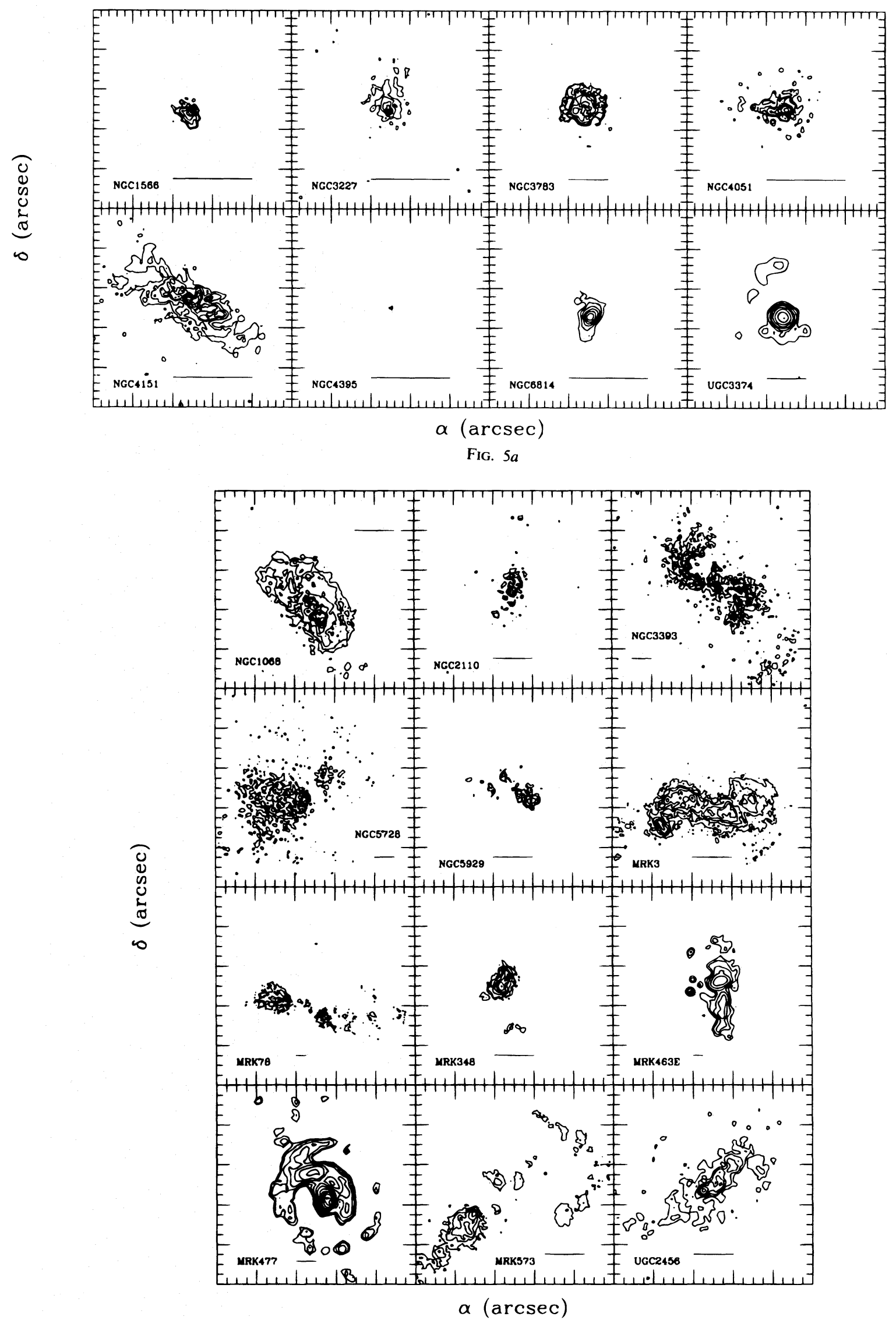

Fig. $5 b$

FIG. 5.-(a) Contour plots of Seyfert 1 galaxies. The upper contour level corresponds to $90 \%$ of the peak of emission, and the following levels correspond to $45 \%, 22.5 \%, \ldots$ of the peak emission. The exception is the lowest contour level, which corresponds to $3 \sigma$ above the sky. The horizontal bar corresponds to $200 \mathrm{pc}$. North is up, and east is to the left. (b) Contour plots of Seyfert 2 galaxies. The lower contour level corresponds to $3 \sigma$, and the horizontal bar corresponds to $200 \mathrm{pc}$. North is up, and east is to the left. 


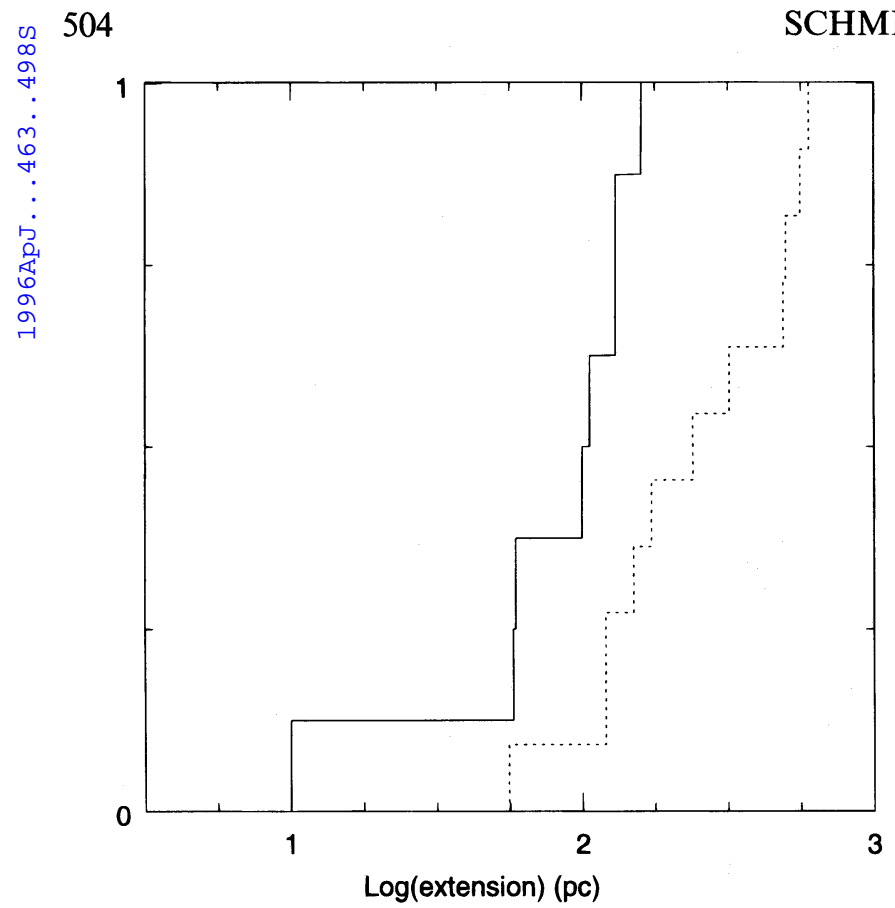

Fig. 6.-Plot of the cumulative distribution functions of the logarithm of the cone base extensions of Seyfert 2 galaxies (dashed line) and Seyfert 1 galaxies (solid line).

Mrk 78 are the exceptions. Because of their large extent, they are displayed with one side corresponding to $4 \mathrm{kpc}$. In Figure 5 we show the contour plots of Seyfert 1's (Fig. $5 a$ ) and Seyfert 2's (Fig. 5b). The lower contour level corresponds to $3 \sigma$ above the sky, and the horizontal bar corresponds to $200 \mathrm{pc}$.

A comparison between our measurements (Table 3 ) and previously published data shows a good agreement. The opening angles of the cones, as well as their position angles, usually agree with the published data within $5^{\circ}$, which is the estimated error in our measurements. However, some gal-

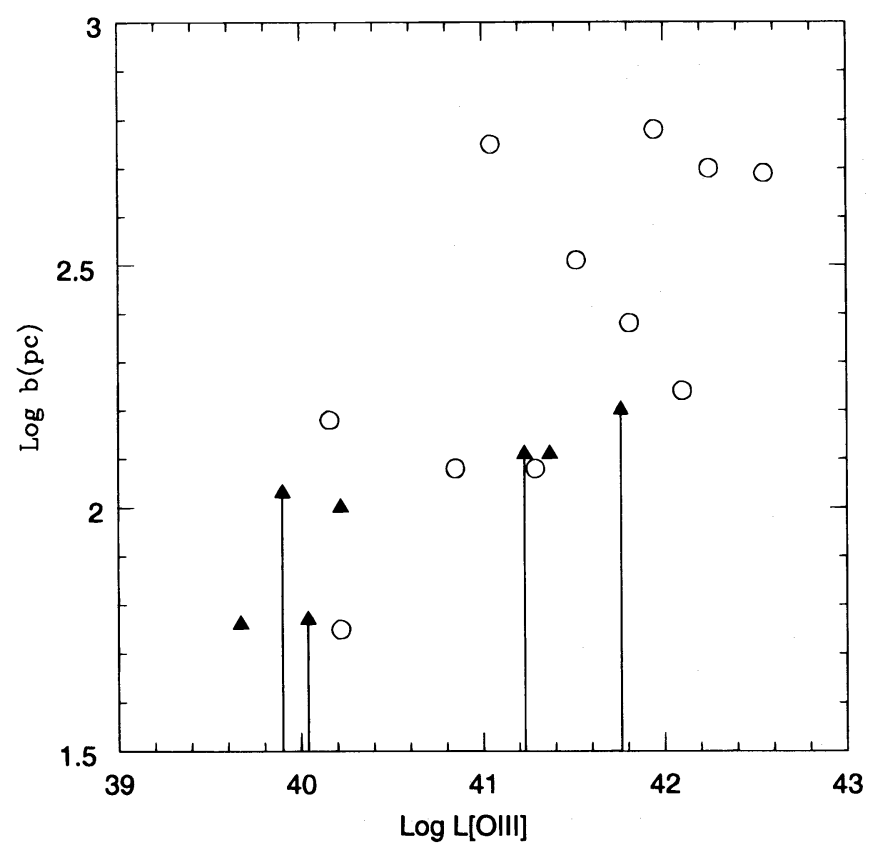

Fig. 7.-Plot of the logarithm of the cone's base extension $(b)$ vs. the logarithm of the [O III] luminosity. Filled triangles are Seyfert 1 galaxies, and open circles are Seyfert 2 galaxies. axies such Mrk 3 and Mrk 573 present slightly different values. Mrk 573 was measured by Pogge \& De Robertis (1993) using lower resolution ground-based data, which resulted in a smaller opening angle, while Capetti et al. (1995) found a much larger opening angle for Mrk 3 because of the fact that they included all the Z-shaped NLRs in their measurements, which we avoided in ours. Our values of the maximum extension of the cone $(d)$ are consistent with published values, although they are slightly smaller because of the conservative measurement technique used.

In order to determine whether the two groups of galaxies are drawn from the same parent population, we show in Figure 6 the cumulative distribution functions of the logarithm of the cone's base extensions ( $b$ ) of Seyfert 1's and Seyfert 2's. As can be seen, the two groups of galaxies differ, with the Seyfert 2's having larger " $b$ " sizes than the Seyfert 1's. Applying a Kolmogorov-Smirnov (K-S) test to the data, we find that the hypothesis that the two groups of galaxies are drawn from the same parent population is rejected at the $95.3 \%$ level. If we apply the K-S test without the Seyfert 1 galaxy NGC 4395, which can be considered an exception, because of the fact that it is the lowest luminosity Seyfert 1 galaxy known, the above hypothesis is still rejected at the $93.7 \%$ level.

The above results apparently contradict the Unified Scheme, from which we would expect " $b$ " to be equal for Seyfert 1 and Seyfert 2 galaxies. One possible explanation for the systematic difference in size is that the obscuring torus axis in the Seyfert 1 galaxies may lie close to the galaxy plane axis, approximately perpendicular to the disk, so that the ionizing continuum shines out of the galaxy and very little gas is available to be ionized. In a similar fashion, the obscuring torus axis in the Seyfert 2 galaxies may lie at an intermediate angle between the galaxy plane axis and the galaxy plane so that there is ample gas available for ionization.

Is it necessary for all the Seyfert 1 host galaxies to be seen face-on, in order for the scenario proposed above to work? Assuming that the average torus opening angle is $\approx 60^{\circ}$, which was obtained by Osterbrock \& Shaw (1988) from the relative number of Seyfert 1 to Seyfert 2 galaxies, and assuming that the mismatch between the galaxy plane axis and the torus axis is as large as $30^{\circ}$, the host galaxy inclination angle relative to the plane of the sky could be as high as $\approx 60^{\circ}$ and still allow the nucleus to be detected as a Seyfert 1 nucleus. An inspection of the inclination angle of the Seyfert 1 galaxies in our sample (Table 2) shows that they are all smaller than $50^{\circ}$, which agrees with the above estimates.

We can calculate the Seyfert 1's cone base extension $(b)$ under this scenario. For this we assume that the torus opening angle is $60^{\circ}$. We assume that the galaxy disk has a thickness of the order of $100 \mathrm{pc}$ in the nuclear region, which is compatible with the fact that spiral galaxies usually have small amounts of $\mathrm{H}_{\mathrm{I}}$ around the nucleus (Rots 1975; Begeman 1988). We find that " $b$ " is $\approx 60 \mathrm{pc}$ in the case in which the torus and the galaxy plane axis are aligned; " $b$ " is $\approx 90 \mathrm{pc}$ in the case in which the torus and the galaxy plane are misaligned by $30^{\circ}$. These values are similar to the measured values.

However, our result is only suggestive, not conclusive, because of the limitations of the sample used. This archival sample is not carefully matched by isotropic properties so 
as to compare objects of similar luminosity. In Figure 7 we show the logarithm of the cone's base extension versus the logarithm of the [O III] luminosity (log $b$ vs. $\log L[\mathrm{O}$ III]). The unresolved base extensions of the Seyfert 1's are shown as upper limits. While this plot suggests that the Seyfert 2's have, as a class, larger NLRs than Seyfert 1's, it is clear that a sample chosen so as to match isotropic luminosities is necessary to show conclusively an intrinsic difference between the NLRs of Seyfert 1's and Seyfert 2's.

While the above suggestion would explain the small NLRs of the Seyfert 1's, it appears inconsistent with observations of extended NLRs (ENLRs), in some Seyfert 2's, such as NGC 1365, NGC 3281, and NGC 7582 (StorchiBergmann \& Bonatto 1991; Storchi-Bergmann, Wilson, \& Baldwin 1992). These galaxies have ionization cones perpendicular to the major axes of their host galaxies extending as much as $2 \mathrm{kpc}$ from the nucleus. However, these galaxies also show outflow of gas in the ENLRs (Hjelm \& Lindblad 1996; Storchi-Bergmann et al. 1992; Morris et al. 1985), which suggests that the mechanism responsible for the production of the ENLRs may be different than the mechanism responsible for production of the NLRs.

\section{SUMMARY}

In this paper we present high-resolution narrow-band [O III] images of Seyfert galaxies, available in the HST Data Archive. The total sample includes eight Seyfert 1's and 12 Seyfert 2's. An analysis of the properties of the two groups of galaxies (distance, [O III] luminosity, and limit of detectability of emission in the images) shows that they have a good overlap of intrinsic properties, which assures us that we are not biased toward the detection of faint emission in Seyfert 2 galaxies.

The comparison between the sizes of the Seyfert 1 NLRs with the sizes that the Seyfert 2 NLRs would have if they were observed pole-on (quantity " $b$ " in Fig. 1) shows that the Seyfert 1's differ considerably from the Seyfert 2's, inconsistent with the Unified Scheme. We propose that a possible explanation for this problem is that the Seyfert 1's have their obscuring torus preferentially aligned perpendicular to the galaxy disk, implying that the ionizing continuum shines out of the galaxy and very little gas is available to be ionized.

We would like to thank R. White and M. Giavalisco for their suggestions about the pre-COSTAR image deconvolution, D. Calzetti for a critical reading of the manuscript, and J. Godfrey for help with the cartoon. This work was supported by the Brazilian institution $\mathrm{CNPq}$ and by NASA through grant number AR-05810.01-94A. This research has made use of the NASA/IPAC Extragalactic Database (NED) which is operated by the Jet Propulsion Laboratory, Caltech, under contract with National Aeronautics and Space Administration.

\section{APPENDIX A}

\section{DESCRIPTION OF INDIVIDUAL OBJECTS}

\section{A1. NGC 1068}

Pogge (1988a) observed that the high-excitation emission is cone shaped and lies along the radio jet observed by Wilson \& Ulvestad (1982). Higher resolution images were obtained by Macchetto et al. (1994), using FOC (not the images presented here), which confirm the presence of the high-ionization cone. The [O III] emission of this galaxy is extended by 7".5 (600 pc) in PA $35^{\circ}$. The nuclear emission has a conical shape with opening angle $45^{\circ}$. Several condensations can be seen inside the cone. The extended emission is coincident with the radio emission shown by Wilson \& Ulvestad (1982). The cone's base extension is $3^{\prime \prime}(240 \mathrm{pc})$. See Evans et al. (1991) for more details about these images. See Macchetto et al. (1994) for details on the images obtained after COSTAR.

\section{A2. NGC 1566}

The WF/PC 1 images of this galaxy have been studied by Kriss et al. (1990). The emission-line image of this Seyfert 1 galaxy is pointlike, having a maximum extent of 0 .'7 $(59 \mathrm{pc})$.

\section{A3. NGC 2110}

Mulchaey et al. (1994) present the [O III] WF/PC 1 observations included in the present work, showing a component extended by $1^{\prime \prime}$ north of the nucleus and an S-shaped ionized gas extended by 4 ". Their [O III] image roughly resembles the radio map. NGC 2110 was observed through a medium-band filter that allowed the transmission of [O III] $\lambda \lambda 4959,5007$ and $\mathrm{H} \beta . \mathrm{H} \beta$ is responsible for $16 \%$ of the total emission in the image, according to the line ratios of Whittle (1992) and the filter transmission curve. We can see that the extended emission of NGC 2110 is curved, having a hook shape. It starts in the nucleus and extends $00^{\prime \prime} 7(97 \mathrm{pc})$ along the northwest direction, then bends toward the northeast and extends $00^{\prime \prime} 6(83 \mathrm{pc})$ in that direction. The total extent of the emission in the north direction is $0.9(125 \mathrm{pc})$. Assuming a conservative measure of the opening angle of the ionization cone, which excludes the curved emission to the northeast, we derive an opening angle of $35^{\circ}$. Some emission is detected at 1" (140 pc) southeast of the nucleus and 2".7 (375 pc) north. The cone's base extension is 0 ".4 (56 pc). For more details on this galaxy, see Mulchaey et al. (1994).

\section{A4. NGC 3227}

Mundell et al. (1995) detected extended [O III] emission in their ground-based data of size $7^{\prime \prime}$ and PA $30^{\circ}$. Their radio maps show a double source separated by 0.4 with PA $-10^{\circ}$, not aligned to the [O III] emission. The HST image presented here shows that this galaxy has a compact nucleus and extended emission with PA $15^{\circ}$. There is a second knot of emission $\approx 0$ ".25 $(18 \mathrm{pc})$ from the nucleus that may be related to the double-peaked radio source found by Mundell et al. (1995) but that is 
extended with PA $-10^{\circ}$. The bulk of the emission is extended by $0.9(64 \mathrm{pc})$ with PA $15^{\circ}$, or 1".4 (100 pc) if we consider the faint emission extending farther north. However, the HST image does not show the extended emission described by Mundell et al. (1995) on ground-based observations, probably because of the very long exposure time of Mundell et al.

A5. NGC 3393

The extended [O III] emission of this galaxy is S-shaped, extending $5^{\prime \prime}(1140 \mathrm{pc})$ along PA $55^{\circ}$. The emission extends $1^{\prime \prime} 8$ $(410 \mathrm{pc})$ on either side of the nucleus, along the east-west direction. The emission then bends toward north and south, following the curvature of the spiral arms. There is also some faint emission 4".5 $(1030 \mathrm{pc})$ southwest of the nucleus. A conservative measurement of the cone opening angle gives $40^{\circ}$ with base extension of $1.4(320 \mathrm{pc})$.

\section{A6. NGC 3783}

An unresolved nuclear point source is detected in radio observations (Ulvestad \& Wilson 1984b), ground-based observations (Winge et al. 1992), and the WF/PC 1 [O III] images presented here (Kinney et al. 1992). The emission-line image of this galaxy is pointlike, with no evidence of extended emission. The total extent of the emission is 0.8 (130 pc). Residuals of the deconvolution are apparent in the image.

\section{A7. NGC 4051}

The [O III] emission of this Seyfert 1 galaxy is unresolved from ground-based observations (Haniff, Wilson, \& Ward 1988; Pogge 1989). Ulvestad \& Wilson (1984a) obtained VLA radio maps that show that this galaxy has two components separated by 0 ."4 along PA $90^{\circ}$. Our image shows an unresolved nucleus and some extended emission with a blob displaced by 0 ."5 (24 pc) from the nucleus with PA $100^{\circ}$. This structure is similar to the radio structure found by Ulvestad \& Wilson (1984a). The total extent of the emission is $1.2(58 \mathrm{pc})$.

\section{A8. NGC 4151}

Pogge (1989) and Pérez et al. (1989) detected [O III] emission in ground-based images that is extended by $\approx 20^{\prime \prime}$ along PA $228^{\circ}$. The [O III] emission was later confirmed to be a biconical structure by WF/PC 1 images (Evans et al. 1993). The [O III] emission of this galaxy has a biconical morphology along PA $55^{\circ}$. The total extent of the emission is $\approx 5^{\prime \prime} .5(350 \mathrm{pc}), 3^{\prime \prime}$ along the southwest direction and 2".5 along the northeast. The total opening angle of the cones is $70^{\circ}$. The base extension is $2{ }^{\prime \prime} .05$ $(130 \mathrm{pc})$, which is not atypical for Seyfert 2 galaxies (in spite of the fact that this is a Seyfert 1 galaxy). For more details, see Evans et al. (1993).

\section{A9. NGC 4395}

This is the least luminous Seyfert 1 galaxy known $\left(M_{B}=-9.8\right.$; Filippenko \& Sargent 1989). Filippenko, Ho, \& Sargent (1993) obtained FOS spectra and WF/PC 1 images centered on narrow-band images. The UV spectrum resembles those of Seyfert 1's, and the narrow-band images are unresolved. The nuclear emission is unresolved with a maximum extent of 0 ".5 $(10 \mathrm{pc})$.

\section{A10. NGC 5728}

Pogge (1989) obtained ground-based [O III] images of this galaxy and found extended $\left(\approx 10^{\prime \prime}\right)$, biconical [O III] emission, with PA $130^{\circ}$. This was confirmed by Wilson et al. (1993) based on the WF/PC 1 images presented here. This galaxy was observed with a medium-band filter that transmitted both [O III] $\lambda \lambda 4959,5007$ and $\mathrm{H} \beta . \mathrm{H} \beta$ contributes $7.5 \%$ of the total emission in the image. Our [O III] image also shows a biconical morphology but with PA $120^{\circ}$. The total opening angle of the cone is $70^{\circ}$, and it is extended by $4^{\prime \prime}(720 \mathrm{pc})$ along the southeast direction and 1"2 $(220 \mathrm{pc})$ into the northwest. Wilson et al. (1993) claimed to see some emission extended by 8 ".5 to the southeast of the nucleus, on the WF/PC 1 images. There is a slight difference between their final images of NGC 5728 and our final images of the same data, due perhaps to the number of iterations in the deconvolution process. The cone's base extension is 3".1 (560 pc). See Wilson et al. (1993) for more details.

\section{A11. NGC 5929}

Bower et al. (1994) obtained the WF/PC 1 images of this object centered on [O III $]$ and $\mathrm{H} \alpha+[\mathrm{N}$ II] that show a conical structure and a dust lane aligned perpendicular to the radio lobes. This galaxy was observed through a medium-band filter that included [O III] $\lambda \lambda 4959,5007$ and $\mathrm{H} \beta$, with $\mathrm{H} \beta$ responsible for $19 \%$ of the total emission in the image. The emission has a cone shape along PA $245^{\circ}$, with opening angle $80^{\circ}$ and total extent of $1^{\prime \prime}(175 \mathrm{pc})$. The opposite side of the nucleus also contains some blobs of emission at a distance of 1".4 (245 pc). The cone's base extension is 0".9 (150 pc). See Bower et al. (1994) for more details.

\section{A12. NGC 6814}

Pogge (1989) found that the nuclear [O III] emission of this galaxy is unresolved when observed from the ground. This galaxy has an unresolved core and some extended emission along PA $150^{\circ}$. The total extent of the emission is $\approx 1^{\prime \prime}(106 \mathrm{pc})$. There is no emission related to the western radio extension found by Ulvestad \& Wilson (1984a), but the overall distribution of the $[\mathrm{O}$ III $]$ emission seems to follow that of the radio emission. 


\section{A13. MRK 3}

This galaxy was observed with a medium-band filter that included $\mathrm{H} \beta$ and $[\mathrm{O}$ III] $\lambda \lambda 4959,5007$, with $\mathrm{H} \beta$ being responsible for $7.5 \%$ of the total emission. The emission-line distribution is Z-shaped, extending $\approx 2$ ". $1(555 \mathrm{pc})$ along the east-west direction. Note the presence of a biconical morphology along PA $70^{\circ}$. The opening angle of the western cone is $40^{\circ}$, and the emission is extended by $1^{\prime \prime} .4(370 \mathrm{pc})$ in that direction, while the eastern cone has an opening angle of $50^{\circ}$ and the emission is extended by $0.7(185 \mathrm{pc})$. The base extension is $0.65(175 \mathrm{pc})$. See Capetti et al. (1995) for more details on the prerefurbishment FOC observations of this galaxy.

\section{A14. MRK 78}

This Seyfert 2 galaxy was observed with FOC by Capetti et al. (1994). Mrk 78 has extended [O III] emission, aligned to the radio axis along PA $84^{\circ}$ (Ulvestad, Wilson, \& Sramek 1981). This galaxy was observed with a medium-band filter that included $\mathrm{H} \beta$ and [O III] $\lambda \lambda 4959,5007, \mathrm{H} \beta$ being responsible for only $6 \%$ of the total emission. The [O III] image shows a biconical emission-line region extended along $\approx 4^{\prime \prime}(3000 \mathrm{pc})$ with PA $70^{\circ}$. The opening angle of the cones is $45^{\circ}$. The optical emission is similar in shape to the radio emission (Ulvestad et al. 1981). The cone's base extension is 0"8 (500 pc). See Capetti et al. (1994) for more details on these prerefurbishment FOC observations.

\section{A15. MRK 348}

This galaxy was observed with a medium-band filter that includes both [O III] $\lambda \lambda 4959,5007$ and $\mathrm{H} \beta . \mathrm{H} \beta$ contributes to $8.5 \%$ of the total emission in the image. The [O III] emission is composed of two blobs separated by $00^{\prime \prime} 15$ (45 pc) along PA $150^{\circ}$, with the nucleus close to the northern component and the total extent of the emission being $0^{\prime \prime} 6(180 \mathrm{pc})$ along this direction. There is also some faint emission at $0.9(270 \mathrm{pc})$ south of the nucleus, but it does not appear to be correlated with the nuclear emission. Assuming that the southern emission has a conical shape, the opening angle is measured to be $60^{\circ}$. The cone's base extension is $0.4(120 \mathrm{pc})$.

\section{A16. MRK 463E}

Uomoto et al. (1993) discovered a jet extended by 0".84 south of the nucleus on these WF/PC 1 [O III] images. They suggest from the photometry of the source that the jet may be the reflecting mirror of the hidden Seyfert 1 nucleus, responsible for the polarization seen by Miller \& Goodrich (1990). This interactive galaxy has a redshift such that the narrow-band filter $(\mathrm{F} 517 \mathrm{~N})$ used to observe it included only the [O III] $\lambda 4959$ and $\mathrm{H} \beta$, while the medium-band filter (F547M) included [O III] $\lambda \lambda 4959$, 5007 and $\mathrm{H} \beta$. Because of this fact, it is not possible to subtract the continuum image from the emission-line image. We can see in the image a compact nucleus and a jetlike structure (Uomoto et al. 1993), extending $\approx 0^{\prime \prime} 9(885 \mathrm{pc}$ ) toward the south. The radio and the optical morphologies of Mrk 463E are similar, extending along the same direction, but not matching each other closely. If we assume that the jet has a conical morphology, we can measure an opening angle $40^{\circ}$. The cone's base extension is 0".5 (490 pc). See Uomoto et al. (1993) for more details.

\section{A17. MRK 477}

Like Mrk 463E, this galaxy has a redshift such that the [O III] $\lambda \lambda 4959,5007$ lines were transmitted through the narrowband (F517N) and the medium-band (F547M) filters. It was also observed through the F785LP filter, which can be used as a continuum filter for comparison with the emission-line images but which cannot be used for subtraction because of the wavelength difference between them. The [O III] emission of this galaxy is complex, with a compact nucleus and a small blob of emission at $0^{\prime \prime} .1(74 \mathrm{pc})$ to the south. Along PA $35^{\circ}$ there is a larger blob separated by $0.4(296 \mathrm{pc})$ from the nucleus and a second blob separated by 0.6 (445 pc). It is also possible to observe some emission to the west of the nucleus, which, together with the emission to the northeast, forms an incomplete ring around it. Farther out, at $\approx 0$ 0"7 $(520 \mathrm{pc})$ from the nucleus, a faint ringlike structure can be seen, which is most likely residuals from the deconvolution. The continuum image is extended along a PA of $35^{\circ}$, showing a compact core, some faint emission around it, and no structure similar to those seen in the emission line images. Because of the complicated morphology of the circumnuclear region, superposition of a cone on the contour plot of this galaxy is difficult.

\section{A18. MRK 573}

The [O III] emission is extended along the same direction as the radio emission (Haniff et al. 1988; Pogge \& De Robertis 1995). This galaxy was observed with a medium-band filter that included [O III] $\lambda \lambda 4959,5007$ and $\mathrm{H} \beta . \mathrm{H} \beta$ is responsible for $8 \%$ of the total emission. The ground-based data show that this galaxy has an extended biconical narrow-line region (Pogge \& De Robertis 1993). The HST image presented here shows the edge of the northwest cone detected by Pogge \& De Robertis (1995); the southeast cone lies outside the detector. The total opening angle of the northwest cone is $70^{\circ}$, and the emission in that direction extends by $2^{\prime \prime}(670 \mathrm{pc})$. The cone's base extension is $1^{\prime \prime} 8(600 \mathrm{pc})$. Note that this measurement includes the northwest blob.

\section{A19. UGC 2456}

This galaxy was observed with a medium-band filter centered on the emission lines [O III] $\lambda \lambda 4959,5007$ and $\mathrm{H} \beta$. $\mathrm{H} \beta$ is responsible for $18 \%$ of the emission in the image. The emission has a jet shape, with some condensations, extended by $1^{\prime \prime} .25$ $(300 \mathrm{pc})$ along PA $310^{\circ}$, similar to the radio jet observed by Ulvestad \& Wilson (1989). Some faint emission can be seen to the southeast of the nucleus, extended by $\approx 0$ " $8(190 \mathrm{pc})$. The cone's base extension is $0.5(120 \mathrm{pc})$. See Bower et al. (1995) for more details on these WF/PC 1 images. 


\section{A20. UGC 3374}

This galaxy was observed through a medium-band filter that had the same transmittance for [O III] $\lambda \lambda 4959,5007$ and $\mathrm{H} \beta$. Using the flux values of Whittle (1992), we can calculate that the $\mathrm{H} \beta$ emission is responsible for $28 \%$ of the total emission in the image. The deconvolution of this galaxy images contained residuals, so that subtraction of the continuum from the emission-line image was impossible. The emission in this object is pointlike, with the bulk of the emission having a maximum extent of 0"4 $(160 \mathrm{pc})$. As Bower et al. (1994) have already pointed out, there is no emission related to the radio lobes observed !. by Ulvestad \& Wilson (1986). See Bower et al. (1994) for more details on the WF/PC images of this galaxy.

Antonucci, R. R. J. 1993, ARA\&A, 31, 473

Antonucci, R. R. J., \& Miller, J. S. 1985, ApJ, 297, 621

Antonucci, R. R. J., \& Ulvestad, J. S. 1985, ApJ, 294, 158

Begeman, K. 1988, Ph.D. thesis, Univ. of Groningen

Bower, G. A., Wilson, A. S., Morse, J. A., Gelderman, R., Whittle, M., \& Mulchaey, J. 1995, ApJ, 454, 106

Bower, G. A., Wilson, A. S., Mulchaey, J. S., Miley, G. K., Heckman, T. M., \& Krolik, J. H. 1994, AJ, 107, 1686

Capetti, A., Macchetto, F., Axon, D. J., Sparks, W. B., \& Boksenberg, A 1995, ApJ, 448, 600

Capetti, A., Macchetto, F., Sparks, W. B., \& Boksenberg, A. 1994, ApJ, 421, 87

de Vaucouleurs, G., de Vaucouleurs, A., Corwin, H. G., Jr., Buta, R. J., Paturel, G., \& Fouqué, P. 1991, Third Reference Catalogue of Bright Galaxies (Berlin: Springer)

Díaz, A. I., Prieto, M. A., \& Wamsteker, W. 1988, A\&A, 195, 53

Evans, I. N., Ford, H. C., Kinney, A. L., Antonucci, R. R. J., Armus, L., \& Caganoff, S. 1991, ApJ, 369, L27

Evans, I. N., Tsvetanov, Z., Kriss, G. A., Ford, H. C., Caganoff, S., \& Koratkar, A. P. 1993, ApJ, 417, 82

Filippenko, A. V., Ho, L. C., \& Sargent, W. L. W. 1993, ApJ, 410, L75

Filippenko, A. V., \& Sargent, W. L. W. 1989, ApJ, 342, L11

Haniff, C. A., Wilson, A. S., \& Ward, M. J. 1988, ApJ, 334, 104

Heckman, T. M. 1995, ApJ, 446, 101

Hjelm, M., \& Lindblad, P. O. 1996, A\&A, 305, 727

Jedrzejewski, R. I., Hartig, G., Jakobsen, P., Crocker, J. H., \& Ford, H. C. 1994, ApJ, 435, L7

Kinney, A. L., Antonucci, R. R. J., Ward, M. J., Wilson, A. S., \& Whittle, M. 1991, ApJ, 377, 100

Kinney, A. L., Kriss, G. A., Ford, H. C., Evans, I. N., Uomoto, A., Caganoff, S., \& Tsvetanov, Z. 1992, BAAS, 24, 727

Kriss, G. A., et al. 1990, BAAS, 22, 1280

Krist, J. 1992, The TINY TIM User's Manual (Baltimore: Space Telescope Science Institute)

Lucy, L. B. 1994, AJ, 79, 745

Macchetto, F., Capetti, A., Sparks, W. B., Axon, D. J., \& Boksenberg, A. 1994, ApJ, 435, L15

MacKenty, J. W., et al. 1992, Wide Field and Planetary Camera Instrument Handbook Version 3.0 (Baltimore: Space Telescope Science Institute)
Morris, S., Ward, M., Whittle, M., Wilson, A. S., \& Taylor, K. 1985, MNRAS, 216, 193

Mulchaey, J. S., Wilson, A. S., Bower, G. A., Heckman, T. M., Krolik, J. H., \& Miley, G. K. 1994, ApJ, 433, 625

Mundell, C. G., Holloway, A. J., Pedlar, A., Meaburn, J., Kukula, M. J., \& Axon, D. J. 1995, MNRAS, 272, 355

Osterbrock, D. E., \& Shaw, R. A. 1988, ApJ, 327, 89

Pérez, E., González-Delgado, R., Tadhunter, C., \& Tsvetanov, Z. 1989, MNRAS, 241, 31P

Pogge, R. W. 1988a, ApJ, 328, 519 $1988 \mathrm{~b}, \mathrm{ApJ}, 332,702$

1989, ApJ, 345, 730

Pogge, R. W., \& De Robertis, M. M. 1993, ApJ, 404, 563

. 1995, ApJ, 451, 585

Rots, A. H. 1975, A\&A, 45, 43

Schmitt, H. R., \& Storchi-Bergmann, T. S. 1995, MNRAS, 276, 592

Schmitt, H. R., Storchi-Bergmann, T. S., \& Baldwin, J. A. 1994, ApJ, 423 237

Sparks, W. B., Ritchie, C., \& MacKenty, J. 1992, WF/PC Instrument Science Report 92-09, Absolute Efficiency of the WF/PC (Baltimore: Space Telescope Science Institute)

Storchi-Bergmann, T., \& Bonatto, C. J. 1991, MNRAS, 250, 138

Storchi-Bergmann, T., Wilson, A. S., \& Baldwin, J. A. 1992, ApJ, 396, 45

Ulvestad, J. S., \& Wilson, A. S. 1984a, ApJ, 278, 544

. 1984b, ApJ, 285, 439

1986, MNRAS, 218, 71

1989, ApJ, 343, 659

Ulvestad, J. S., Wilson, A. S., \& Sramek, R. A. 1981, ApJ, 247, 419

Uomoto, A., Caganoff, S., Ford, H. C., Rosenblat, E. I., Antonucci, R. R. J., Evans, I. N. \& Cohen, R. D. 1993, AJ, 105, 1308

Urry, C. M., \& Padovani, P. 1995, PASP, 107, 803

Winge, C., Pastoriza, M. G., Storchi-Bergmann, T., \& Lipari, S. 1992, ApJ, 393,98

Whittle, M. 1992, ApJ, 79, 49

Wilson, A. S., Braatz, J. A., Heckman, T. M., Krolik, J. H., \& Miley, G. K. 1993, ApJ, 419, L61

Wilson, A. S. \& Ulvestad, J. S. 1982, ApJ, 263, 576

Wilson, A. S., Ward, M. J., \& Haniff, C. A. 1988, ApJ, 334, 121 